\title{
Comparing Solar Minimum 23/24 with Historical Solar Wind Records at 1 AU
}

\author{
L.K. Jian · C.T. Russell · J.G. Luhmann
}

Received: 23 October 2010 / Accepted: 18 February 2011 / Published online: 1 April 2011

(C) The Author(s) 2011. This article is published with open access at Springerlink.com

\begin{abstract}
Based on the variations of sunspot numbers, we choose a 1-year interval at each solar minimum from the beginning of the acquisition of solar wind measurements in the ecliptic plane and at $1 \mathrm{AU}$. We take the period of July 2008 -June 2009 to represent the solar minimum between Solar Cycles 23 and 24. In comparison with the previous three minima, this solar minimum has the slowest, least dense, and coolest solar wind, and the weakest magnetic field. As a result, the solar wind dynamic pressure, dawn-dusk electric field, and geomagnetic activity during this minimum are the weakest among the four minima. The weakening trend had already appeared during solar minimum $22 / 23$, and it may continue into the next solar minimum. During this minimum, the galactic cosmic ray intensity reached the highest level in the space age, while the number of solar energetic proton events and the ground level enhancement events were the least. Using solar wind measurements near the Earth over 1995 - 2009, we have surveyed and characterized the large-scale solar wind structures, including fast-slow stream interaction regions (SIRs), interplanetary coronal mass ejections (ICMEs), and interplanetary shocks. Their solar cycle variations over the 15 years are studied comprehensively. In contrast with the previous minimum, we find that there are more SIRs and they recur more often during this minimum, probably because more low- and mid-latitude coronal holes and active regions emerged due to the weaker solar polar field than during the previous minimum. There are more shocks during this solar minimum, probably caused by the slower fast magnetosonic speed of the solar wind. The SIRs, ICMEs, and shocks during this minimum are generally weaker than during the previous minimum, but did not change as much as did the properties of the undisturbed solar wind.
\end{abstract}

The Sun-Earth Connection near Solar Minimum

Guest Editors: M.M. Bisi, B.A. Emery, and B.J. Thompson.

L.K. Jian ( $\varangle) \cdot$ C.T. Russell

Institute of Geophysics and Planetary Physics, University of California, Los Angeles, CA 90095, USA

e-mail: jlan@ igpp.ucla.edu

J.G. Luhmann

Space Science Laboratory, University of California, Berkeley, CA 94720, USA 


\section{Introduction}

It is well known to the solar and space physics community that the solar minimum between the Solar Cycles 23 and 24 has been unusually long and deep. There have been a number of studies on this solar minimum as it developed (see, e.g., McComas et al., 2008; Smith and Balogh, 2008; Gibson et al., 2009; Lee et al., 2009). A series of conferences and workshops were also held focusing on the solar minimum, e.g., the Whole Heliosphere Interval (WHI) Workshops, the SOHO-23 Workshop (Cranmer, Hoeksema, and Kohl, 2010, as the conference proceeding). Now that the new activity of Solar Cycle 24 has risen, it is a good time to look back and define the interval of the real solar minimum. In Section 2, we use over 40 years of measurements to compare solar minimum 23/24 with previous minima during the space age, to achieve a more comprehensive understanding of this solar minimum and also to appreciate the variability of solar activity, solar wind, resultant cosmic ray and geomagnetic behaviors.

Besides the basic solar wind parameters, the large-scale solar wind structures are also important and some of them can be geoeffective. These structures include the stream interaction region (SIR, in nature the same as the term co-rotating interaction regions used by many others) formed when a fast solar wind overtakes and interacts with the preceding slow solar wind (see, e.g., Gosling and Pizzo, 1999), the interplanetary counterpart of coronal mass ejection (ICME), and the interplanetary shock often driven by an ICME or SIR. Continuing the long-term study of these structures at 1 AU (Jian et al., 2006a, 2006b), we have surveyed these structures at 1 AU over 1995-2009, using the in-situ observations from Wind and Advanced Composition Explorer (ACE) spacecraft near the Sun-Earth Lagrange point L1. In Section 3, we compare the features of these large-scale solar wind structures during this solar minimum 23/24 with those during the previous minimum. We do not include solar minimum $21 / 22$ in the comparison, because the solar wind measurements taken then are not complete and have too many data gaps for us to correctly identify and characterize the solar wind structures.

\section{Solar Minimum 23/24 in the Context of Space Age}

\subsection{Solar Activity and Solar Magnetic Field}

Sunspot number (SSN) is often treated as a primary indicator of solar activity. Figure 1(a) illustrates the variation of 27-day averaged SSN from November 1963 to November 2010. The green dashed line, marking the SSN of 25, is added to indicate the low-level solar activity. At this writing, the SSN has finally reached 25, so the period of SSN below 25 at this minimum is longer than four years. To treat the solar minima consistently, we choose a 1-year period around the smallest SSN to represent each solar minimum, as denoted by the magenta bars in Figures 1-5. From these figures, we can see that, although different parameters reach their solar-minimum extremes at different times, these times nearly all fall in the 1-year intervals. On the other hand, some of the parameters vary significantly within 1 year from the smallest SSN time. Hence, a 1-year period is an appropriate length to represent each minimum and we do not only focus on a couple of WHI Carrington rotations (CRs) in this paper.

For solar minimum 23/24, the selected time period is 1 July 2008 - 30 June 2009, which is better illustrated in Figure 2(a) using the daily SSN and logarithmic scale. There are 

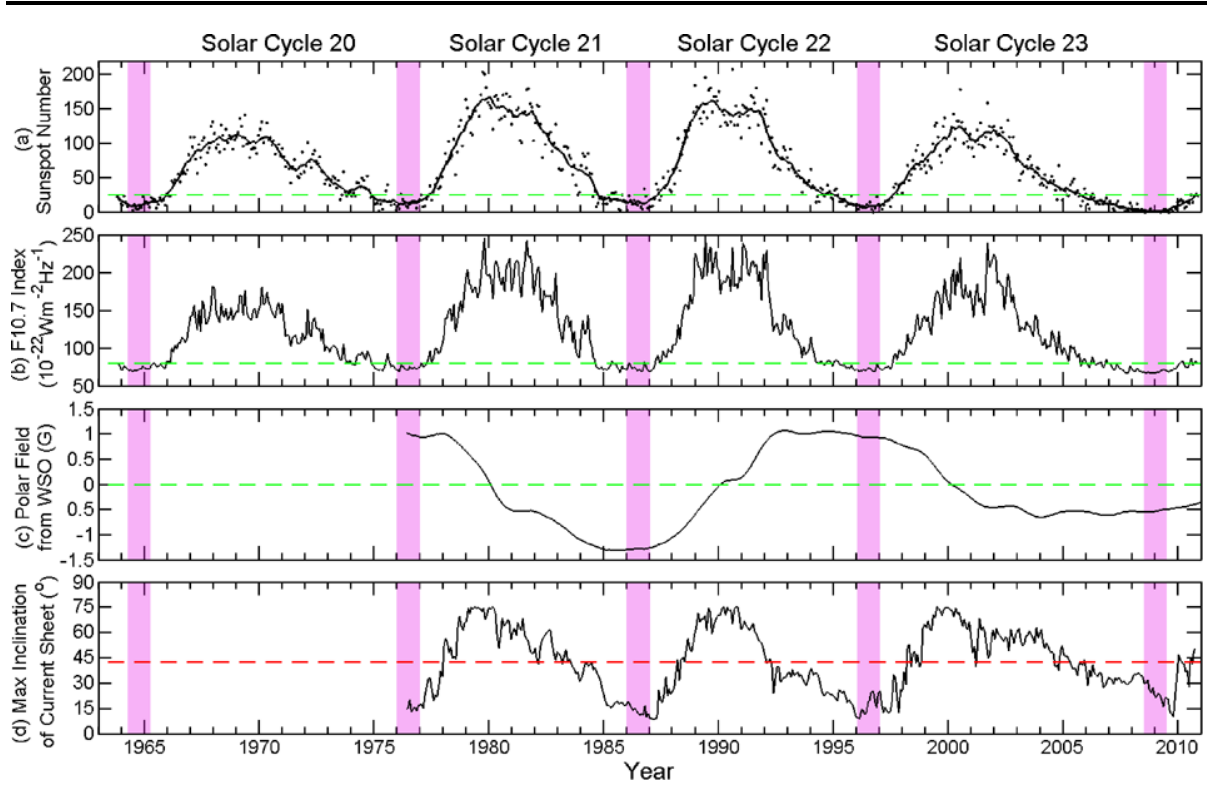

Figure 1 Variations of solar background since the early time of the space era. (a) Dots: 27-day averages of SSN from NOAA's National Geophysical Data Center (NGDC); black solid line: 11-point running average. (b) Black solid line: 27-day average of the F10.7 index from NGDC, indicating the solar radio flux at a wavelength of $10.7 \mathrm{~cm}$ at the Earth orbit. (c) Black solid line: 10-day average of solar polar field (North and South) over May 1976-December 2010 from the WSO site (http://wso.stanford.edu/Polar.html). A 20 nHz low-pass filter is used to eliminate yearly geometric projection effects. (d) Black solid line: computed maximum inclination of the HCS for each Carrington rotation from the WSO site (http://wso.stanford.edu/Tilts.html); red dashed line: average inclination over the whole data set. The magenta bars indicate the 1-year solar minimum intervals used for quantitative comparison.

294 spotless days during this 1 -year period, covering about $80 \%$ of the time, and the 27 day averaged SSN is only $1.9 \pm 0.4$, where the uncertainty is the probable error of the mean. The SSN of this minimum is significantly smaller than during the minimum 22/23 $(8.5 \pm 1.5)$ and the previous minima $20 / 21$ and $21 / 22$ as shown in Figure 2(a). In addition, we note that solar minima $20 / 21$ and $21 / 22$ have more SSNs than the minima 22/23 and 23/24, and the following Cycles 21 and 22 have more SSNs overall than Cycle 23. It is predicted that Solar Cycle 24 will be a weak cycle with maximum SSN smaller than 60 (http://solarscience.msfc.nasa.gov/images/ssn_predict_l.gif). Since SSN relates to magnetic flux emergence (Gibson, Webb, and Thompson, 2010), from the above correlation, we can speculate that the solar magnetic flux at the solar minimum strongly modulates the SSN and strength of the following cycle.

Besides SSN, the F10.7 index is also an important gauge of solar activity and often used in ionospheric models to represent the solar radiation. This index is measured as the solar radio flux per unit frequency at a wavelength of $10.7 \mathrm{~cm}$, which is near the peak of the observed solar radio emission. Because the emission at the centimetric wavelength is mainly due to the coronal plasma trapped by magnetic fields over active regions, the F10.7 index can indicate the overall solar activity levels. As shown in Figure 1(b), the F10.7 index during solar minimum 23/24 is smaller than during the previous four solar minima, suggesting overall weaker solar activity.

Since May 1976, the Wilcox Solar Observatory (WSO) has used its polemost 3' apertures to measure the solar polar field strength (Svalgaard, Duvall, and Scherrer, 1978; Murdin, 
(a) Daily Sunspot Number

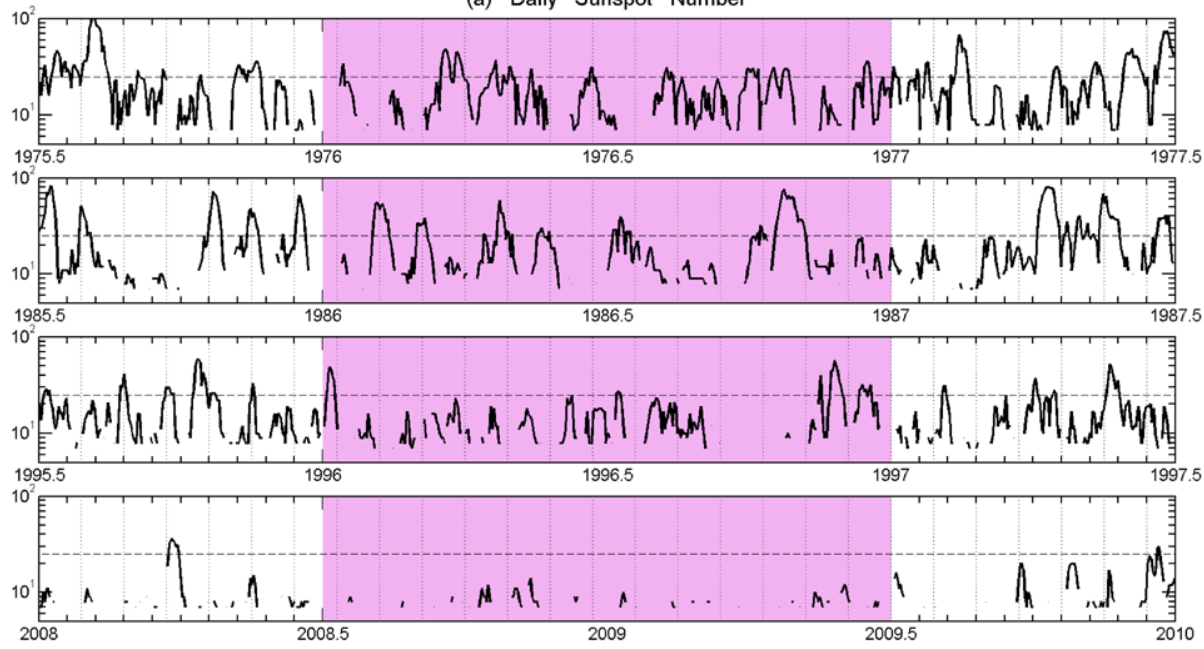

(b) Latitude of Coronal Field Neutral Line $($ )
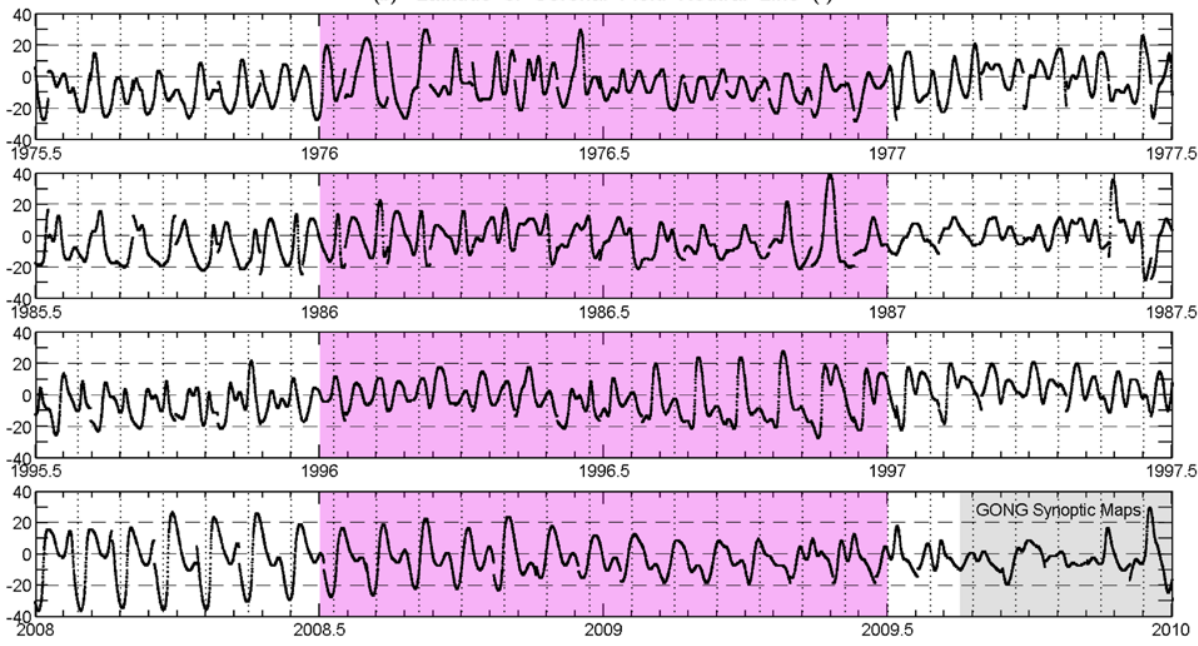

Figure 2 The variations of SSN and neutral line latitude over two years of each solar minimum for the four latest solar minima. The vertical dotted lines separate the 27.3-day solar rotations. The start time of each rotation is arbitrary not CR time. The magenta bars indicate the 1-year period of each minimum. The daily international SSN is from the Solar Influences Data Analysis Center in Belgium (http://sidc.oma.be/). Because the logarithmic scale is used, the spotless days are shown as no data. The horizontal dashed line marks SSN of 25. The latitude of coronal field neutral line is obtained using the PFSS model. The synoptic maps for the inner boundary of the model are from the MWO all the time except 2009.63-2010, when GONG maps are used, as denoted by the gray bar in the last panel.

2000). Figure 1(c) displays the variation of the solar polar field over three solar cycles, averaging measurements at the North and South, with the yearly geometric projection effects removed. In comparison with Solar Cycles 21 and 22, the field strength did not significantly increase in 2004, the middle of the declining phase. Instead, the field remained weak for five years, with a gradually decreasing trend in the recent years. The polar field strength during solar minimum $23 / 24$ is only $0.527 \pm 0.004$ Gauss, about $56 \%$ of that during the previous minimum $(0.936 \pm 0.002 \mathrm{G})$ and $41 \%$ of that during solar minimum $21 / 22(1.280 \pm$ 
$0.001 \mathrm{G})$. Using the polar faculae on the white-light images obtained at the Mount Wilson Observatory (MWO, Ulrich et al., 2002) since 1906, Sheeley (2010) concluded that the polar fields are weaker now than they have been in the last 100 years. From the interplanetary magnetic field (IMF) measurements which will be addressed in Section 2.2, we can ascertain that the weak magnetic field is not a characteristic of only the polar regions, but of the whole Sun (McComas et al., 2008; Lee et al., 2009; Luhmann et al., 2009).

The "traditional" picture of the solar minimum emphasizes the idea of a dipolar corona and outflows from polar coronal holes that diverge to fill the interplanetary space, so the heliospheric current sheet (HCS) is roughly in the solar equatorial plane (Hundhausen, 1972). Associated with the weaker solar field during solar minimum 23/24, the HCS morphology differs from previous minima too. Figure 1(d) illustrates the maximum inclination of the HCS for each CR since May 1976, obtained from the WSO website (Hoeksema, 1995; and references therein). This inclination indicates the envelope of the HCS neutral line warp, and it is computed using a "classic" potential field source surface (PFSS) model applied to the photospheric magnetic field observed by the WSO, with the source surface at 2.5 solar radii (Schatten, Wilcox, and Ness, 1969; Altschuler and Newkirk, 1969; Hoeksema, Wilcox, and Scherrer, 1983). This model assumes that the photospheric field has a meridional component and requires a polar field correction to more closely match the observed IMF structure at Earth. In the long declining phase of Solar Cycle 23, the HCS became less warped more gradually than in previous two solar cycles, which is likely attributed to the weakness of the polar fields (Wang et al., 2010). The average latitudinal extent of the HCS during solar minimum $23 / 24$ is $24^{\circ} \pm 1^{\circ}$, larger than during the previous two minima. Similar to Solar Cycle 22, which was one Hale cycle back, the HCS of Solar Cycle 24 started with a flat geometry and rapidly evolved to more warped shape at the end of 2009, but the increasing trend is much faster than before.

To closely compare the coronal field, we plot the latitude of the coronal field neutral line over two years of each solar minimum in Figure 2(b). The neutral line is a good predictor of the overall shape and location of the HCS except that its inclination is often higher in the interplanetary space (Crooker et al., 1996; and references therein). The latitudes are obtained using the PFSS model again with the source surface at 2.5 solar radii. Because of a wildfire in late summer of 2009 , the MWO was temporarily out of operation, and the synoptic maps from the Global Oscillation Network Group (GONG) of the National Solar Observatory (Harvey et al., 1996; Leibacher, 1999) were used as the inner boundary of the PFSS model, from 20 August to the end of 2009, as marked by the gray bar. The different sources of the synoptic maps may bring some inconsistency in the results, but the computed coronal field maps from the WSO (not shown) also confirm that CRs 2087-2089 (20 August -8 November of 2009) are the flattest over the two years. There are more solar rotation periods with the neutral line latitude outside of $\pm 20^{\circ}$ during 2008-2009 than during the previous three minima, again indicating that the coronal field is overall more tilted during this minimum. The HCS flattening and SSN decrease do not occur at the same pace. The flattest HCS over minimum 23/24 did not occur within July 2008 - June 2009, but about two months later, similar to the minimum 21/22. Similar lag between SSN and IMF reaching minimum levels has been found during this minimum and prior minima too (Gibson, Webb, and Thompson, 2010). It may be because SSN relates to the emergence of magnetic flux, while HCS tilt and IMF relate to the evolution of "open" magnetic flux at the Sun (Gibson, Webb, and Thompson, 2010). Nevertheless, we still use the interval July 2008 - June 2009 to represent solar minimum 23/24 because it has the lowest SSN and can be conveniently compared with other solar minima. 


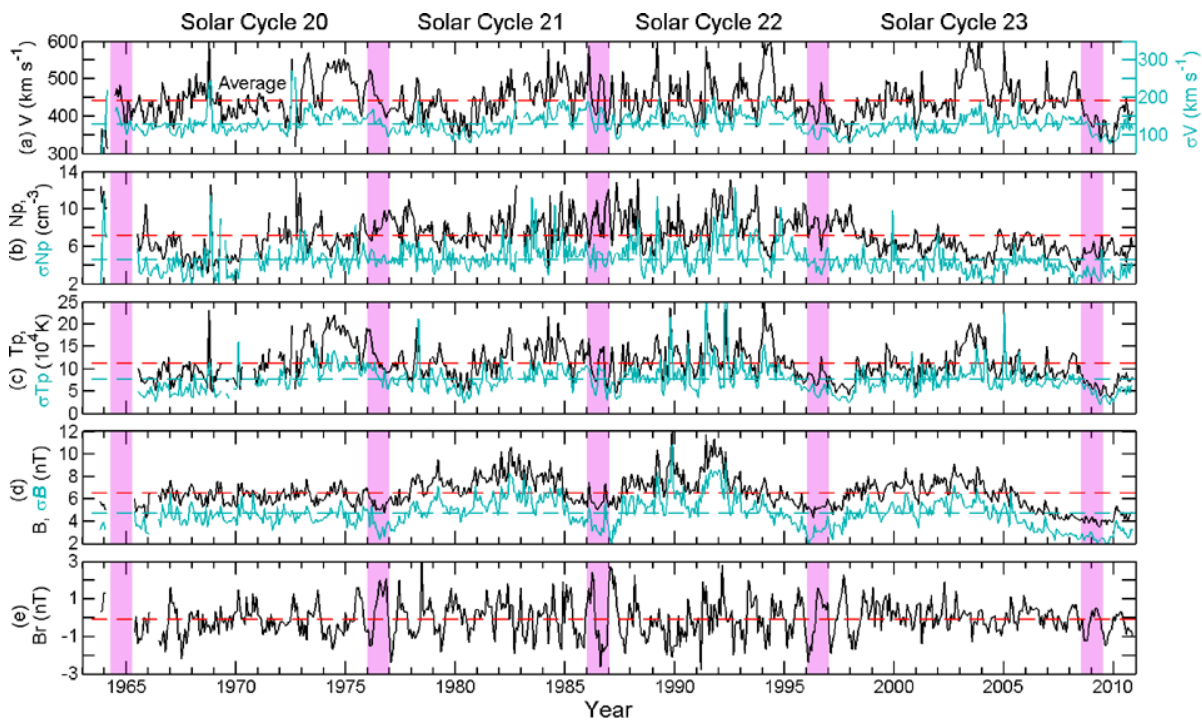

Figure 3 Variations of 27-day averages of solar wind parameters from November 1963 to November 2010: (a) solar wind speed (black) and its standard deviation (teal), (b) proton number density (black) and its standard deviation (teal), (c) proton temperature (black) and its standard deviation (teal), (d) IMF strength and its standard deviation computed as $\left[\left(\sigma B_{x}\right)^{2}+\left(\sigma B_{y}\right)^{2}+\left(\sigma B_{z}\right)^{2}\right]^{1 / 2}$, i.e., the length of the vector formed from the standard deviations of component averages, (e) the radial component of IMF ( + radial outward). These parameters are obtained from the OMNI data base. The standard deviations relate to the 27-day averages and do not capture the variances in the higher resolution data. The red and teal dashed lines mark the overall averages of the corresponding parameters. The magenta bars indicate the 1-year solar minimum intervals used for quantitative comparison in Table 1.

\subsection{Solar Wind Parameters and Geomagnetic Activity Indices}

The Space Physics Data Facility at the Goddard Space Flight Center of NASA provides the near-Earth solar wind plasma and magnetic field measurements to the public through the OMNIWeb (http://omniweb.gsfc.nasa.gov/; King and Papitashvilii 1994, 2005). The lowresolution data (1-hour, 1-day, and 27-day) are available from 1963. The earliest solar wind data were from the Interplanetary Monitoring Platform (IMP) 1 mission. After that, data from more than 10 missions have been processed to generate the OMNI data set. To highlight the long-term variations, we use the 27-day averaged data in Figures 3 and 4. The quantitative comparison of this minimum with previous three minima is given in Table 1, where 12 parameters are listed and the daily data for each minimum are used to obtain better statistics.

In Figure 3(a), the black and teal solid lines denote the solar wind speed and is root-meansquare (RMS) standard deviation (relating to the 27-day average), respectively. The red and teal dashed lines indicate the average speed $\left(442 \mathrm{~km} \mathrm{~s}^{-1}\right)$ over the 46 years and the average of the standard deviation, respectively. Although the solar wind speed varies constantly, it is generally faster in the declining phase of solar cycles, which is attributed to the recurrent fast streams. The 27-day averaged solar wind speed decreased noticeably in 2008 and 2009, from $518 \mathrm{~km} \mathrm{~s}^{-1}$ to $330 \mathrm{~km} \mathrm{~s}^{-1}$, and it finally began to increase in 2010 . The average solar wind speed during this solar minimum is only $388 \pm 4 \mathrm{~km} \mathrm{~s}^{-1}, 8 \%$ slower than during the previous minimum, and $15 \%$ slower than during the minimum 22/23 (Table 1). 


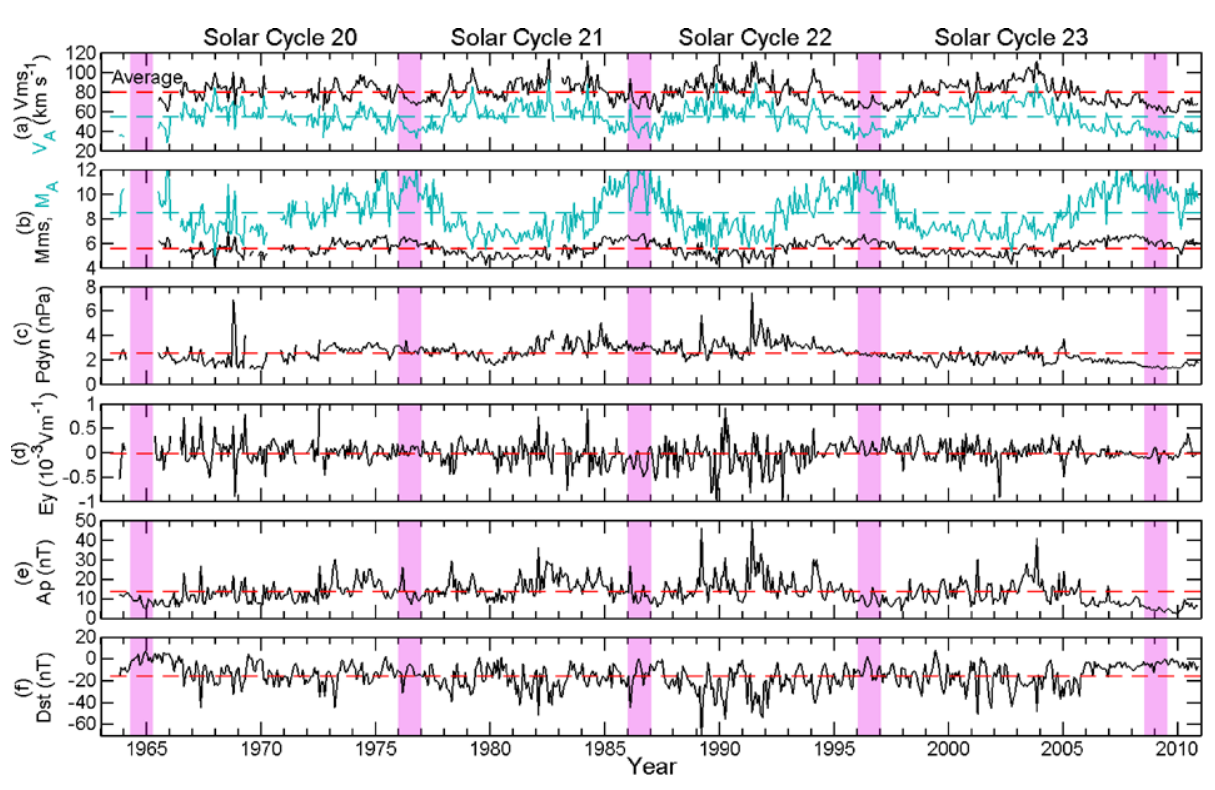

Figure 4 Variations of 27-day averages of solar wind parameters and geomagnetic activity indices since the beginning of the acquisition of solar wind measurements. (a) Fast magnetosonic speed (black) and Alfvén speed (teal). (b) Fast magnetosonic Mach number (black) and Alfvénic Mach number (teal). (c) Dynamic pressure of solar wind where the $\alpha$ particles have been taken into account, assuming $N_{\alpha} / N_{\mathrm{p}}=5 \%$, $T_{\alpha}=4 T_{\mathrm{p}}$, and $V_{\alpha}=V_{\mathrm{p}}$ (http://ftpbrowser.gsfc.nasa.gov/bow_derivation.html). (d) Dawn-dusk component of electric field, positive for duskward, and negative for dawnward. (e) Ap index from NGDC. (f) Dst index from the World Data Center for Geomagnetism, Kyoto (http://wdc.kugi.kyoto-u.ac.jp/index.html). The dashed lines mark the overall averages. The magenta bars indicate the 1-year solar minimum periods used for quantitative comparison.

There were no solar wind proton density records for the solar minimum 19/20. The proton density of Solar Cycles 20 and 23 is generally below the average level of $7.2 \mathrm{~cm}^{-3}$ marked by the red dashed line in Figure 3(b), while the density of Cycles 21 and 22 is generally above the average level. We note the SSNs and F10.7 indices of Solar Cycles 21 and 22 are more than those of Cycles 20 and 23 (Figure 1), suggesting the Solar Cycles 21 and 22 are more active. This may be the cause of the larger density and stronger IMF of the two cycles. The average proton density of solar minimum $23 / 24$ is $5.5 \pm 0.2 \mathrm{~cm}^{-3}$, less than $70 \%$ of that of previous three minima (Table 1). Such small density of solar wind is also obtained from the interplanetary scintillation (IPS) observations (see, e.g., Manoharan, 2009). As displayed in Figure 3(c), the proton temperature of the solar wind varies over the four cycles similarly to the solar wind speed, consistent with the fact that the fast wind is usually hotter than the slow wind. The average proton temperature for this minimum is $64 \pm 3$ kilo-Kelvin, $24 \%$ cooler than during the previous minimum. The steady decreases of solar wind speed and proton temperature in 2008 and 2009 are similar to those during solar minimum 20/21. The RMS standard deviations of proton number density and temperature corresponding to the 27 -day averages, marked by teal solid lines, vary similarly to the variables themselves.

Associated with the lower solar field during this solar minimum, the IMF intensity is about $4.02 \pm 0.08 \mathrm{nT}, 23 \%$ weaker than during the previous minimum, and further weaker than during minima 20/21 and 21/22 (Table 1). As illustrated in Figure 3(d), except for Solar Cycle 20, the IMF usually varies in phase with solar activity, being stronger at the 
Table 1 Comparison of solar wind parameters for four solar minima.

\begin{tabular}{llllllll}
\hline Solar Min Period & $V\left(\mathrm{~km} \mathrm{~s}^{-1}\right)$ & $B(\mathrm{nT})$ & $N_{\mathrm{p}}\left(\mathrm{cm}^{-3}\right)$ & $T_{\mathrm{p}}\left(\times 10^{3} \mathrm{~K}\right)$ & $P_{\mathrm{t}}(\mathrm{pPa})$ & $\beta$ \\
\hline $20 / 21$ & 1976 & $449 \pm 6^{*}$ & $5.56 \pm 0.12$ & $8.2 \pm 0.3$ & $125 \pm 5$ & $43.4 \pm 1.7$ & $3.8 \pm 0.2$ \\
$21 / 22$ & 1986 & $459 \pm 7$ & $5.78 \pm 0.14$ & $8.5 \pm 0.3$ & $109 \pm 5$ & $42.9 \pm 1.4$ & $3.4 \pm 0.2$ \\
$22 / 23$ & 1996 & $422 \pm 4$ & $5.16 \pm 0.08$ & $8.0 \pm 0.2$ & $84 \pm 3$ & $36.0 \pm 0.8$ & $3.5 \pm 0.1$ \\
$23 / 24$ & July 2008 - & $388 \pm 4$ & $4.02 \pm 0.08$ & $5.5 \pm 0.2$ & $64 \pm 3$ & $22.9 \pm 0.7$ & $4.1 \pm 0.3$ \\
& June 2009 & & & & & & \\
\hline Solar Min & Period & $V_{\mathrm{A}}\left(\mathrm{km} \mathrm{s}^{-1}\right)$ & $M_{\mathrm{A}}$ & $V_{\mathrm{MS}\left(\mathrm{km} \mathrm{s}^{-1}\right)} M_{\mathrm{MS}}$ & $P_{\text {dyn }}(\mathrm{nPa})$ & Dst (nT) \\
\hline $20 / 21$ & 1976 & $46.6 \pm 1.1$ & $12.3 \pm 0.3$ & $75.7 \pm 1.1$ & $6.01 \pm 0.05$ & $2.81 \pm 0.07$ & $-13.6 \pm 0.9$ \\
$21 / 22$ & 1986 & $48.0 \pm 1.4$ & $12.0 \pm 0.2$ & $75.2 \pm 1.3$ & $6.18 \pm 0.05$ & $2.97 \pm 0.07$ & $-15.6 \pm 1.0$ \\
$22 / 23$ & 1996 & $42.8 \pm 0.7$ & $12.1 \pm 0.2$ & $69.3 \pm 0.7$ & $6.13 \pm 0.03$ & $2.44 \pm 0.04$ & $-10.9 \pm 0.6$ \\
$23 / 24$ & July 2008- $40.6 \pm 0.8$ & $12.0 \pm 0.2$ & $66.0 \pm 0.7$ & $5.90 \pm 0.03$ & $1.40 \pm 0.03$ & $-4.5 \pm 0.4$ \\
& June 2009 & & & & & & \\
\hline
\end{tabular}

* Probable error of the mean, which is the standard deviation divided by the square root of the event count.

maximum and early declining phase of a solar cycle. Such stronger IMF may be partly due to the fact that more ICMEs occur at these phases and the fields of ICMEs are often stronger than ambient solar wind. The exception of Solar Cycle 20 may have something to do with multiple data sources and data gaps in the early record. Since the IMF is sensitive to the heliocentric distance and spacecraft location, it is more easily affected than other plasma parameters. For instance, as one source of the data of the 1970s, Helios spacecraft had an orbit ranging from 0.3 to $1 \mathrm{AU}$. The solar wind speed and temperature do not vary much with heliocentric distance, but the IMF does. Although such variations have been considered in the OMNI data process, there still could be discrepancies from the in-situ measurements at $1 \mathrm{AU}$. Similar to the proton density, the IMF is stronger in Solar Cycles 21 and 22 than in the other two cycles. The standard deviation of magnetic field vector $(\sigma \mathbf{B}$, teal line) and the standard deviation of field magnitude ( $\sigma B$, not shown) both follow the IMF variation trend, with the former generally larger than the latter. As the IMF changes its direction and intensity frequently, $\sigma \mathbf{B}$ is significant, smaller than the field itself by only about $2 \mathrm{nT}$.

As the radial component of IMF vector presumably represents the unaltered solar field, we illustrate its time variation in Figure 3(e). The magnitude of the 27-day average of the radial field component (taking the sign into the averaging) is stronger during solar minima than during other phases, probably due to less field meandering and fewer transient solar wind structures during solar minima. Consistent with the weaker IMF, the radial field magnitude is noticeably weaker in Solar Cycles 20 and 23, and it is significantly lower in solar minimum 23/24 than in the previous three solar minima. The annual polarity change of $B \mathrm{r}$, named as Rosenberg-Coleman effect (Rosenberg and Coleman, 1969), due to the tilt of solar ecliptic plane with respect to the solar equatorial plane, is pronounced in Figure 3(e). This effect appears to be clearer when the solar activity is weaker.

Figure 4(a) shows the time variations of Alfvén speed (teal line) and fast magnetosonic speed (black line). They roughly follow the variation of IMF, being stronger during solar maxima and early declining phases, indicating the important contribution of IMF to Alfvén speed. Since the sonic speed does not vary much with the solar activity (not shown here), it is no surprise that the fast magnetosonic speed varies similarly to the Alfvén speed. Both fast 
magnetosonic speed and Alfvén speed during this minimum are slower than during previous minima (Table 1), and this can help explain why more interplanetary shocks occurred during this solar minimum than the previous one, which will be addressed in Section 3.4. In Figure 4(b), both the Alfvénic and fast magnetosonic Mach numbers of the solar wind vary in antiphase with solar activity, being largest during solar minima. The values during this solar minimum are not much different from those during previous minima.

The solar wind dynamic pressure and dawn-dusk component of the electric field are two critical parameters that affect the magnetospheric and ionospheric activity of the Earth (see, e.g., Dungey, 1961; Burton, McPherron, and Russell, 1975; Perreault and Akasofu, 1978; Palmroth et al., 2004). Figure 4(c) and (d) illustrate the variations of these two parameters over the last four solar cycles. The dawn-dusk electric field $E_{y}$ is approximated by $-V B_{z}$ in the OMNI data set, where $V$ is the solar wind speed and $B_{z}$ is the IMF north-south component in the geocentric solar magnetospheric (GSM) coordinates. The $E_{y}$ parameter is averaged from hourly electric field data, where the hourly $V$ and $B_{z}$ are used. The dynamic pressure is stronger in Solar Cycles 21 and 22, and weaker in the other two cycles, similar to the variation of proton number density. The average dynamic pressure of this solar minimum is $1.40 \pm 0.03 \mathrm{nPa}$, about only a half of that during the previous minimum. Such a weak dynamic pressure will cause the state, dynamics, and energetics of planetary bow shock and/or magnetosphere during this minimum to be different from other minima (see, e.g., Zhang et al., 2007). The dawn-dusk electric field varies around zero. The dawnward electric field is predominant in Solar Cycles 21 and 22, while the duskward electric field appears more often in Cycles 20 and 23. It is probably related to the Hale-cycle variation of solar magnetic field. There are fewer strong- $E_{y}$ intervals during Solar Cycle 23 than during prior solar cycles, and the absolute value of $E_{y}$ during this minimum is smaller than during previous minima.

Consistent with the weakest dynamic pressure and dawn-dusk electric field of the solar wind, geomagnetic activity indicated by the Ap and Dst indices in Figure 4(e) and (f) is weakest during this solar minimum too. Specifically, the Ap index indicates the changes of magnetic field arising from the Earth's mid-latitude ionospheric current systems. The disturbance storm time index Dst measures the strength of the longitudinally-averaged depression in the geomagnetic field at the equator, whose principal contributor is the ring current (Crooker et al., 1996). Strikingly, the 27-day averaged Dst index has remained above $-20 \mathrm{nT}$ since 2006, indicating the quiet-time ring current in this solar minimum is weaker than in previous minima. Using higher resolution Dst index (hourly and daily), we can verify that there are indeed few big geomagnetic storms in the prolonged solar minimum. Among the four solar cycles, the geomagnetic activity of Cycle 22 is strongest according to the Ap and Dst indices, associated with the strongest dynamic pressure and $E_{y}$ disturbances then.

From the variations of plasma $\beta$ in Table 1 , we can tell that during this minimum the magnetic pressure makes a lesser contribution to the total pressure (the sum of magnetic and plasma thermal pressure) than during previous minima, consistent with the weaker IMF. The total pressure of this minimum is about $36 \%$ smaller than during the previous minimum. In addition, the averages of all the parameters listed in Table 1 except $\beta, M_{\mathrm{A}}$, and $M_{\mathrm{MS}}$ are smaller during the recent two solar minima than those during the minima 20/21 and 21/22; these values during solar minimum 23/24 are smaller than during minimum 22/23. Based on historical proxy data from the early 1800 s, it is possible that such a trend will be followed during the next two solar minima (Russell, Luhmann, and Jian, 2010). Based on the current unusually weak solar magnetic field, Solar Cycle 24 is predicted to be very weak, so it is possible that the speed, density, temperature, and IMF of the solar wind at minimum $24 / 25$ will continue decreasing and become even smaller than during the current minimum. 
Besides the changes of the properties during the four solar minima, we note that the solar radio flux, proton number density, IMF, dynamic pressure, and geomagnetic activity are generally stronger for Solar Cycles 21 and 22, which have more sunspots.

Although the solar wind measurements shown above are taken in the ecliptic plane at $1 \mathrm{AU}$, the differences between the first and third polar orbits of Ulysses also demonstrate that the open magnetic flux is smaller, and the solar wind out of the ecliptic plane is slower, less dense, cooler, and has less mass and momentum flux during solar minimum 23/24 than during the previous minimum (see, e.g., McComas et al., 2008; Smith and Balogh, 2008). Using the 3-D IPS measurements made by the Solar-Terrestrial Environment Laboratory (Kojima and Kakinuma, 1987) of Nagoya University, Tokumaru, Kojima, and Fujiki (2010) reported the significant differences in the solar wind speed distribution closer to the Sun (up to 0.1 AU) between 1996 and 2008. In comparison with 1996, the fast wind areas in 2008 increased at low latitudes and decreased at other latitudes, resulting in a net decrease at all latitudes, consistent with the in-situ solar wind measurements, and such changes are attributed to the weak polar field during this minimum (Tokumaru et al., 2009; Tokumaru, Kojima, and Fujiki, 2010). In addition, using the IPS measurements at the Ooty Radio Telescope, Manoharan (2010) reported the remarkable difference of the solar wind density turbulence during solar minimum 23/24 and suggested that this peculiar solar minimum experienced a low level of coronal density turbulence and the scattering diameter of the corona had decreased steadily after 2003.

\subsection{Solar Energetic Protons and Cosmic Ray Intensity}

When solar activity is strong, solar protons can be accelerated to high energies either close to the Sun by solar flares or in the interplanetary space by shocks. These energetic protons are a significant radiation hazard to spacecraft and astronauts. We use the solar protons with energies greater than $10 \mathrm{MeV}$ from the OMNI data set as an indicator of the radiation variability. As shown in Figure 5(a), the flux of these protons varies in phase with solar activity. There have been no strong energetic proton events since 2007 (see, e.g., Kahler, 2010). The background level of this flux is significantly lower during this solar minimum than during the previous minima.

Another indicator of such radiation variability is the record of the ground level enhancements (GLEs). These enhancements are the sharp increases in the cosmic ray count by at least $10 \%$ recorded by ground-based detectors, and they are often associated with solar energetic proton events. The Australian Antarctic Data Center provides the long-term GLE record, as denoted by teal triangles in Figure 5(b). GLEs occur relatively rarely, several times per solar cycle. There are no GLEs during the five solar minima marked by the magenta bars, except for one event in 1976 of minimum 20/21. As a long solar cycle, Cycle 23 has more solar energetic proton events and more GLEs than the previous cycle. The last GLE event occurred in December 2006. Since then, there have been no GLEs for more than three years.

In contrast, the galactic cosmic ray (GCR) has an easier access to the inner heliosphere during solar minimum so its intensity varies inversely with solar activity. Using the continuous record of cosmic rays from the Oulu Neutron Monitor (http://cosmicrays.oulu.fi/), the cosmic ray intensity reaches the record high during this solar minimum, as illustrated 


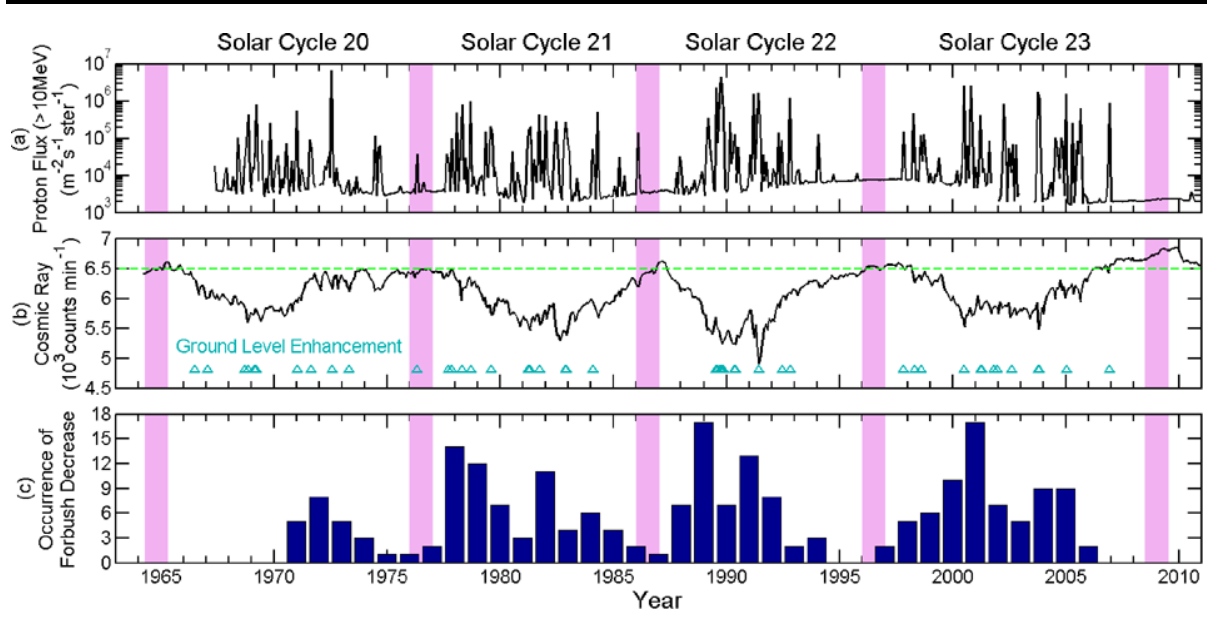

Figure 5 Variations of solar energetic protons and cosmic ray intensity over four solar cycles. (a) 27-day average flux of solar protons of energies higher than $10 \mathrm{MeV}$, obtained from the OMNI data base. (b) Black solid line: cosmic ray intensity from the Oulu Neutron Monitor (http://cosmicrays.oulu.fi/), corrected for both barometric pressure and efficiency; teal triangles: GLE events during 1966-2009 from the World GLE data base at the Australian Antarctic Data Center. (c) Forbush decrease events over 1971-2009 from Oh and Yi (2009) and Oh (personal communication, 2010). The magenta bars indicate the 1-year solar minimum periods the same as Figure 1.

in Figure 5(b). It continued to rise till the end of 2009, and finally dropped in 2010. Such high cosmic ray intensity is also reported by Mewaldt et al. (2010) using ACE observations, where they found an increase of at least $20 \%$ in the intensities of multiple cosmic-ray nuclei from $\mathrm{C}$ to $\mathrm{Fe}$ compared with the previous minimum. As discussed in McDonald, Webber, and Reames (2010) as well as Mewaldt et al. (2010), there are several factors that could cause the space-age cosmic ray maximum during this solar minimum: weaker IMF, reduced IMF turbulence, slower solar wind, and weaker solar wind dynamic pressure than during the previous minima. In addition, the HCS, although not flatter than previous minima, is much flatter than during other phases of Solar Cycle 23. As the HCS warp declined in 2009, shown in Figure 1(d), the cosmic rays finally obtained a more direct inward access than along the earlier wavy HCS.

When GCRs are shielded by the magnetic fields associated with coronal mass ejections (CMEs), neutron monitors detect reductions in the cosmic ray, namely Forbush decrease events (see, e.g., Burlaga, 1991). Such reduction varies from $3 \%$ to $20 \%$, and lasts typically from several hours to a few days. Because Forbush decreases are associated with CMEs, their detection has been used in conjunction with other indications such as the coronal and heliospheric observations to further confirm the presence of CMEs and provide 24-hour advanced warnings of geomagnetic storms. Figure 5(c) plots the histogram distribution of Forbush decrease events over 1971 - 2009 from Oh and Yi (2009) and Oh (personal communication, 2010). They selected these events requiring that the GCR intensity decrease during the main phase to be more than $3 \%$ for all three neutron monitor stations and the minimum intensity of the main phase to be lower than $-1.5 \%$. There were no Forbush decrease events during 2007-2009 (Oh, personal communication, 2010), consistent with the low occurrence rate and weak nature of the CMEs during this minimum which will be addressed in Section 3.3. 


\section{Large-Scale Solar Wind Structures: Solar Minima 22/23 vs. 23/24}

\subsection{Data Set and Identification Criteria}

We have used the in-situ plasma and magnetic field observations from Wind and ACE spacecraft near the Earth to survey the large-scale solar wind structures. Because the Wind mission has a longer operation time, we make the survey using mainly Wind data. But in order to create as complete a survey as possible, when Wind spacecraft is near the Earth's bow shock or magnetosphere, or when it has data gaps or noisy data, we use ACE data. For the Wind spacecraft, we mainly use the 93-second resolution data from the Solar Wind Experiment (SWE, Ogilvie et al., 1995) and the Magnetic Fields Investigation (MFI, Lepping et al., 1995). To identify shocks, 6-12-second resolution SWE data, 3-second resolution MFI data, and 24-second 3-D Plasma Analyzer (Lin et al., 1995) data are used. From the ACE spacecraft, we use the validated Level 2 data from the Solar Wind Electron, Proton, and Alpha Monitor (SWEPAM, McComas et al., 1998) and the Magnetic Field Experiment (Smith et al., 1998), both at the 64-second time resolution. The 16-second magnetic field data are used to identify shocks.

Jian et al. (2006a, 2006b) performed a statistical survey of SIRs and ICMEs over 1995 2004, respectively. Continuing these studies, we use the following criteria to visually identify SIRs: overall solar wind speed increase, pile-up of total perpendicular pressure (Russell, Shinde, and Jian, 2005) with gradual declines on both sides, first increase and then decrease of proton number density, temperature increase, entropy change, solar wind flow deflection, and IMF compression associated with field shear (see, e.g., Gosling and Pizzo, 1999; Jian et al., 2006a; and references therein). With low coverage of temperature anisotropy data, we assume that the temperature is isotropic, so the total perpendicular pressure is essentially the same as the total pressure. From here, we will use the term "total pressure" $P_{\mathrm{t}}$. Because not all the SIR features appear for every SIR, we require five of them, with the speed increase as one necessary criterion. To uniformly define the stream interface, we choose the time when the total pressure peaks as the stream interface (Jian et al., 2006a).

To identify ICMEs, we use the following characteristics: stronger than ambient magnetic field, relatively quiet field, smooth rotations of magnetic field, declining solar wind speed, low proton temperature, high $\alpha$ particle abundance, low $\beta$, enhanced total pressure, bidirectional suprathermal electron strahl (see, e.g., Gosling, 1997; Jian et al., 2006b; Zurbuchen and Richardson, 2006; Richardson and Cane, 2010; and references therein). Because none of these features is unique to ICMEs, we require at least three signatures to be an ICME. To ensure the identification of some ambiguous events, we have inspected the coronal and heliospheric observations for possible CME sources too.

Our surveys have been served as Level 3 contributed data at the ACE Science Center (http://www.srl.caltech.edu/ACE/ASC/DATA/level3/index.html). Because different research groups have different understandings and emphases to multiple signatures of SIRs and ICMEs, our survey can be different from other catalogs. For example, having a higher weight on the magnetic field and plasma features of the ICME, our ICME list has some discrepancy from the list of Richardson and Cane (2010). It would be good for the readers to consult multiple independent surveys when needed.

Using the high-resolution plasma and magnetic field data, we have identified interplanetary shocks associated with these SIRs or ICMEs, which are marked in the surveys. For interested readers, there are other surveys of interplanetary shocks to refer to, for instance, the ones from the Interplanetary Shock Database at the HarvardSmithsonian Center for Astrophysics (http://www.cfa.harvard.edu/shocks/), the ACE team 

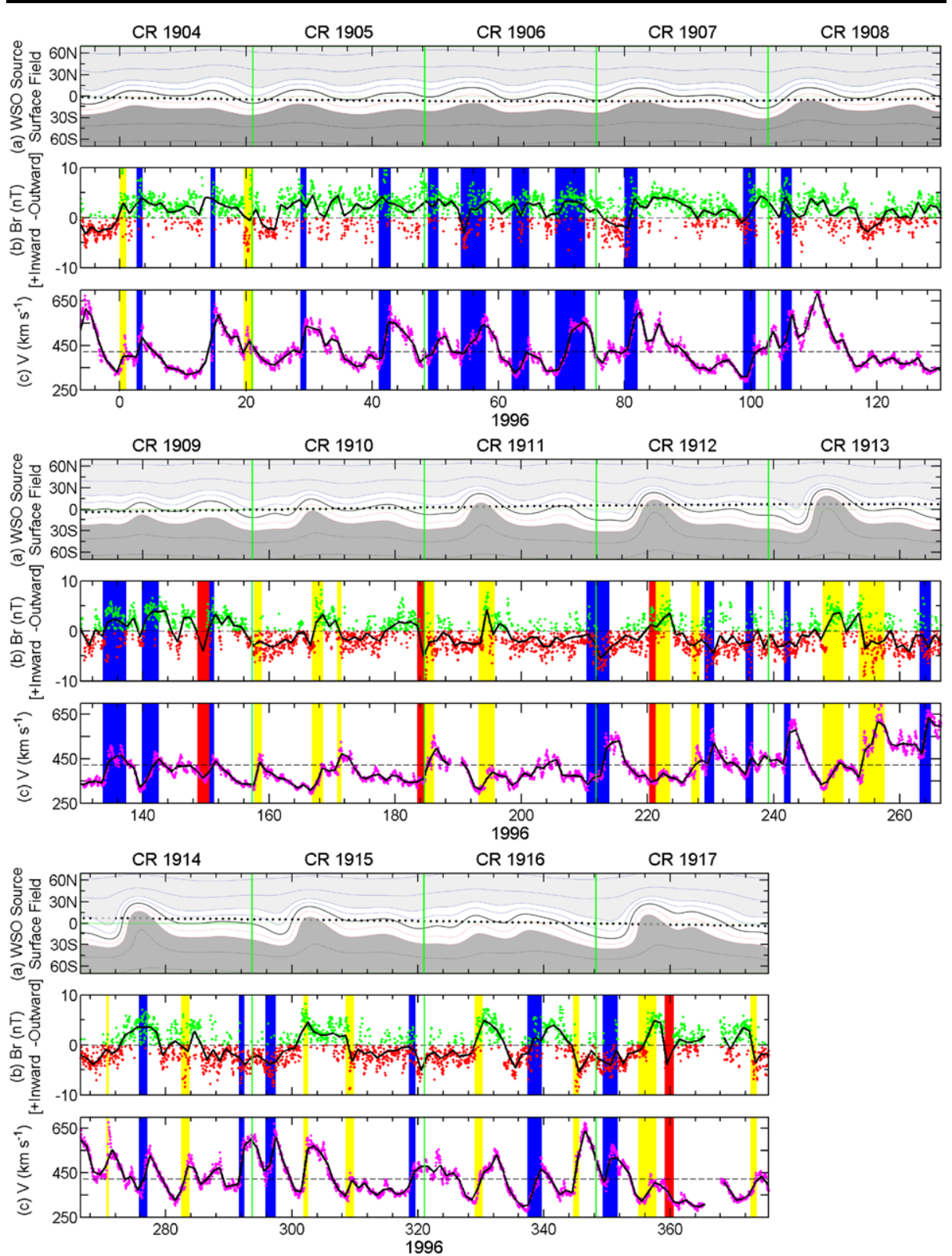

Figure 6 The coronal field and solar wind stream structures in 1996, during solar minimum 22/23. From top to bottom: (a) the coronal field at the source surface 2.5 solar radii using the classic computation from the WSO (http://wso.stanford.edu/synsourcel.html), (b) the radial component of IMF, (c) the solar wind speed. The CR numbers at the top refer to panel (a), the day numbers at the bottom refer to panels (b) and (c). Panels (b) and (c) have 4-day solar wind propagation time lag from panel (a). In (a), the light and dark gray regions indicate opposite field polarities; the black curved line denotes the coronal field neutral line; and the black dotted line marks the heliographic latitude of the Earth. In (b) and (c), the dots are hourly OMNI data, and the lines are daily OMNI data. The green and red dots in (b) represent radial inward and outward IMF, respectively. The black dashed line in (c) marks the average solar wind speed over the 14 CRs. The green vertical lines separate the CRs. The red vertical bars indicate the ICMEs; the yellow bars denote the SIRs with a HCS crossing within three days of the stream interface; and the blue bars mark the SIRs not associated with any HCS crossing. 
(http://www.bartol.udel.edu/ chuck/ace/ACElists/obs_list.html\#shocks), and Berdichevsky et al. (2000).

\subsection{Stream Interaction Regions: Solar Minima $22 / 23$ vs. $23 / 24$}

Figures 6 and 7 display the comparison of the coronal field and solar wind stream structures between solar minima $22 / 23$ and $23 / 24$, respectively. The green vertical lines separate the CRs. The panel (a) shows the coronal field at the source surface of 2.5 solar radii obtained from the WSO (Schatten, Wilcox, and Ness, 1969; Altschuler and Newkirk, 1969; Hoeksema, Wilcox, and Scherrer, 1983). The panels (b) and (c) display the radial component of IMF and the solar wind speed. Considering the solar wind propagation time, we have shifted these two panels forward by four days. Using the changes of the IMF polarity and the suprathermal electron pitch angle distribution (not shown here), we identify the HCS crossings, requiring that either side of the crossing has a uniform field direction for longer than three days. The yellow vertical bars denote the SIRs associated with a HCS crossing within three days of the stream interface, while the blue vertical bars indicate the SIRs without any HCS crossing nearby. The red bars mark the ICMEs.

As illustrated in Figures 6 and 7, the solar wind during the solar minima lacks a consistent pattern in the time series. Gibson et al. (2009) noticed this for solar minimum 22/23, but they did not choose the end of 2008 as the center of solar minimum 23/24, because the real minimum had not been established yet at that time. The solar wind streams vary irregularly, different from the recurring well-defined fast streams often pictured for the declining phase. Using the 3-D IPS observations, Bisi et al. (2010) also found the velocity co-rotation became less dominant than the declining phase and the Sun's dipole-like structure in velocity became more complex (see, e.g., Bisi et al., 2009). The fast wind is not always fast, sometimes even slower than $400 \mathrm{~km} \mathrm{~s}^{-1}$. Although the polar coronal holes appear smaller during the minimum 23/24 than during the previous one (Kirk et al., 2009), there are a few long-lasting coronal holes in the low- and mid-latitude regions (see, e.g., Abramenko et al., 2010), as additional sources of fast wind. Therefore, within one CR, there were multiple fast solar wind streams and multiple resultant SIRs which meet our SIR criteria, not only the speed increase criterion. CRs 1906, 1912, 2075, and 2078 are such examples. Although some of the SIRs have unusually slow fast stream and small speed increase, the variations of their velocity vector, density, temperature, and other parameters clearly demonstrate they are SIRs.

In Figures 6 and 7, the sub-Earth location is indicated by the black dotted line in panel (a). It is obvious that there is no one-to-one correlation between the coronal field neutral line crossing and the HCS crossing at $1 \mathrm{AU}$. Neither is there a one-to-one correlation between the coronal field neutral line crossing and the SIR at 1 AU. For instance, in early CR 1908, the coronal field neutral line crossing was not associated with any HCS crossing at $1 \mathrm{AU}$; in the second half of CR 2073, the coronal field neutral line crossing and HCS crossing were not related to any SIR at 1 AU. During the solar minima, although the coronal field changed gradually over one solar rotation, a few SIRs did not recur after one CR. Some of the SIRs associated with the HCS crossings (yellow bars) recurred for several solar rotations and remained the association with HCS crossing. For example, the second SIR of CR 1910 recurred for eight times, and the association with HCS crossing remained except for CR 1914. Not all SIRs associated with HCS crossings recurred. A recurring SIR can be associated with HCS crossing at one solar rotation, and not at the next solar rotation, e.g., the SIR at the beginning of the CR 1911. A HCS can be missed when the spacecraft is a little off to one side of the HCS. In addition, from the backward PFSS mapping, we note 

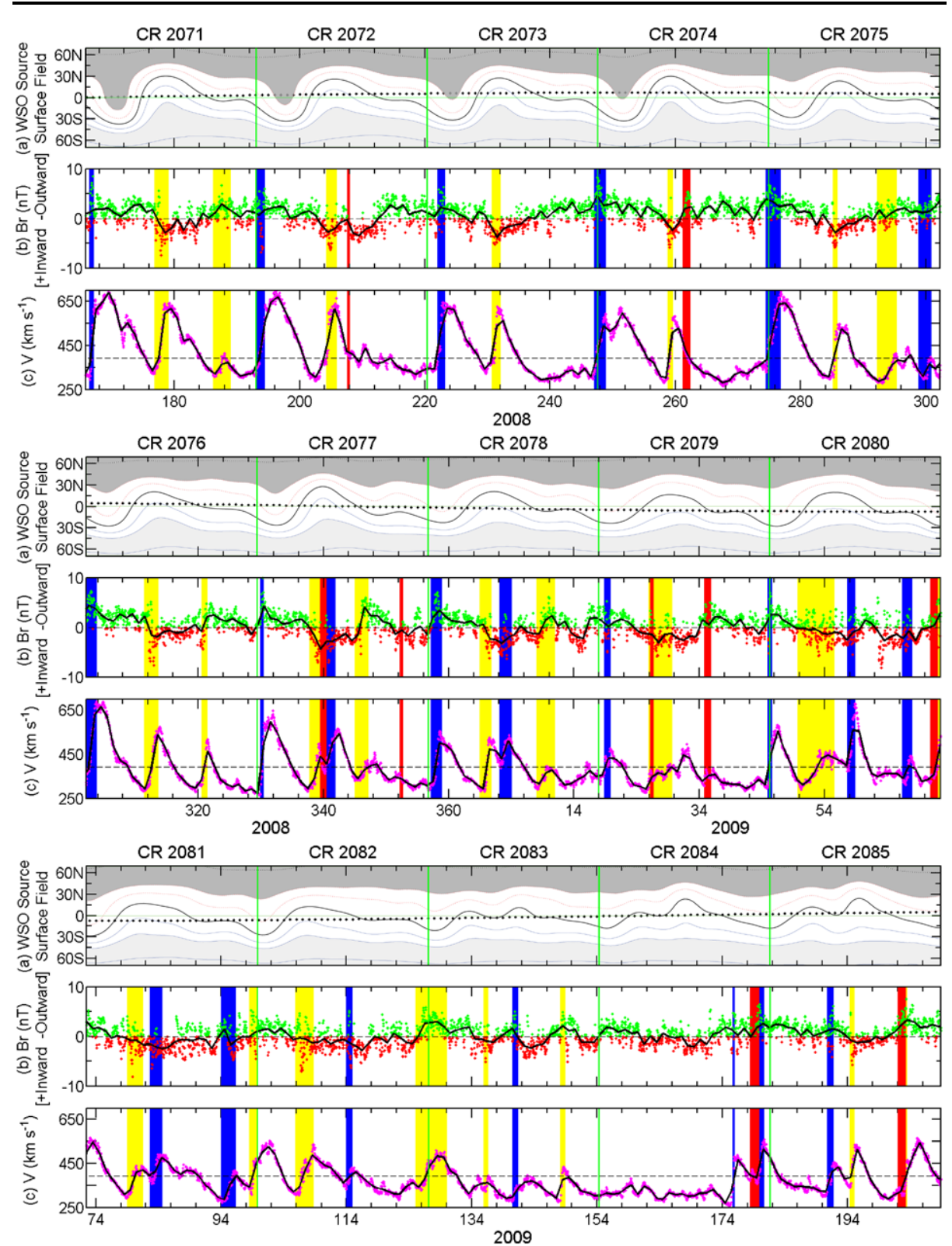

Figure 7 The coronal field and solar wind stream structures during solar minimum 23/24. The captions of Figure 6 apply.

the SIRs associated with HCS crossings are not necessarily connected to the polar coronal holes. The small coronal holes or active regions in the northern and southern hemispheres can have different polarities, and the SIRs associated with them will be adjacent to the IMF polarity changes and the resultant HCS crossings. 
Table 2 Comparison of SIRs between solar minima 22/23 and 23/24.

\begin{tabular}{|c|c|c|c|c|c|c|c|}
\hline Solar Min & $\begin{array}{l}\text { SIR } \\
\text { Number }\end{array}$ & \multicolumn{2}{|c|}{$\begin{array}{l}\text { SIRs Associated with } \\
\text { HCS Crossings } \\
\text { (Rate) }\end{array}$} & $\begin{array}{l}\text { Recurring- } \\
\text { SIR Number } \\
\text { (Rate) }\end{array}$ & $\begin{array}{l}\text { Shock } \\
\text { Number } \\
\text { (Rate) }\end{array}$ & $\begin{array}{l}\text { Duration } \\
\text { (hour) }\end{array}$ & Size (AU) \\
\hline $22 / 23$ & 42 & $17(40 \%)$ & & $37(88 \%)$ & $7(17 \%)$ & $44 \pm 4$ & $0.32 \pm 0.03$ \\
\hline $23 / 24$ & 41 & $21(51 \%)$ & & $39(95 \%)$ & $16(39 \%)$ & $43 \pm 4$ & $0.30 \pm 0.03$ \\
\hline Solar Min & $\begin{array}{l}V_{\max } \\
\left(\mathrm{km} \mathrm{s}^{-1}\right)\end{array}$ & $\begin{array}{l}V_{\min } \\
\left(\mathrm{km} \mathrm{s}^{-1}\right)\end{array}$ & $\begin{array}{l}V_{\text {mean }} \\
\left(\mathrm{km} \mathrm{s}^{-1}\right)\end{array}$ & $\begin{array}{l}\Delta V \\
\left(\mathrm{~km} \mathrm{~s}^{-1}\right)\end{array}$ & $B_{\max }(\mathrm{nT})$ & $\begin{array}{l}P_{\mathrm{t} \max } \\
(\mathrm{pPa})\end{array}$ & $\begin{array}{l}P_{\text {dynmax }} \\
(\mathrm{nPa})\end{array}$ \\
\hline $22 / 23$ & $554 \pm 12$ & $349 \pm 6$ & $451 \pm 8$ & $205 \pm 9$ & $12.8 \pm 0.4$ & $129 \pm 7$ & $7.2 \pm 0.5$ \\
\hline $23 / 24$ & $525 \pm 15$ & $320 \pm 5$ & $423 \pm 9$ & $203 \pm 13$ & $12.0 \pm 0.6$ & $114 \pm 10$ & $6.2 \pm 0.6$ \\
\hline
\end{tabular}

Table 2 compares SIRs during the two solar minima. In comparison with solar minimum $22 / 23$, the SIRs during the minimum $23 / 24$ occur at a similar rate, with a higher recurrent rate, probably because the solar background is quieter at this minimum. About half of the SIRs during this minimum are associated with HCS crossings, slightly more often than during the previous minimum, but both lower than the 15-year average rate of $57 \%$ marked by the green dotted line in Figure 8(c). In addition, we have compared the average values of the duration, size, maximum speed, minimum speed, mean speed, speed enhancement, maximum magnetic field strength, maximum total pressure, and maximum dynamic pressure of the SIRs during the two minima in Table 2. The size is computed as the product of the mean speed, duration, and the sine of the spiral angle, and it is roughly the projected width perpendicular to the stream interface in the solar equatorial plane (see Jian et al., 2008 for more detail). The average properties of SIRs are generally weaker during this minimum than during the previous minimum. However, if we take the uncertainty into account, all these SIR parameters during the two solar minima do not differ much, except that the maximum, minimum, and mean speeds of SIRs during solar minimum 23/24 are slower. The slower fast wind is consistent with the fact that coronal holes inside a belt of $\pm 40^{\circ}$ around the solar equator occupied more area during this minimum than during the previous minimum (Abramenko et al., 2010).

To illustrate the solar cycle variations of SIR features, we plot their annual averages along with the SSN from 1995 to 2009 in Figure 8. The annual event number of SIRs denoted by the white bars varies from 32 to 45 over the 15 years, being smallest in solar maximum and slightly larger in the declining phase and solar minima. The cyan bars indicate the CIRs, which recur at least once. The recurrent rate is exceptionally high in 2007 and 2008, and is generally lower around solar maximum. During the two solar minima, about $90 \%$ of the SIRs recur (Table 2), which is consistent with the relatively steady solar background. The HCS association rate of SIRs is generally higher in the declining phase and in 1999. The rate is especially low in 1996 and 2009, near 40-45\%. The size, mean speed, and speed increase of SIRs in the declining phase are generally larger than at any other time. In 2009, the annual averages of the mean speed, speed increase, maxima of total pressure and dynamic pressure all reach the lowest values over the 15 years. Comparing the averages in Table 2 with the green dotted lines in Figure 8, we can see that the mean speed, speed increase, maximum $P_{\mathrm{t}}$, and maximum dynamic pressure during the solar minima 22/23 and 23/24 are all smaller than the 15-year average.

So far, we have not used a speed criterion for our SIR identification. If we require the fastest speed of the SIR to be above 450 or $500 \mathrm{~km} \mathrm{~s}^{-1}$, the event number will decrease 
Figure 8 The variations of the annual average SIR properties from 1995 to 2009. From top to bottom: (a) monthly SSN from NOAA, (b) SIR (white) and CIR association rate, (d) size, (e) mean solar wind speed of a SIR, (f) speed increase over a SIR, (g) maximum total pressure, (h) maximum dynamic pressure. The error bar indicates the probable error of the mean. The red dashed line denotes the median value over all the SIRs of the 15 years, while the green dotted line for the 15-year average value. Extended after Jian et al. (2006a). (cyan) event number, (c) HCS
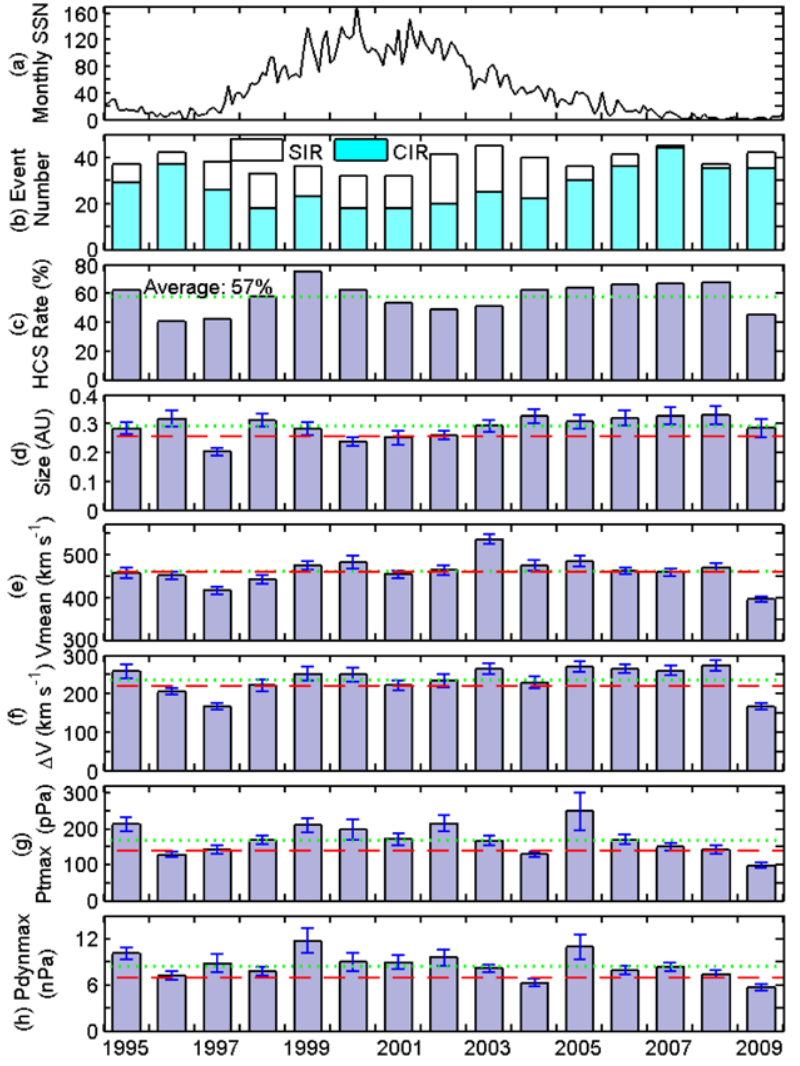

Figure 9 The number of events from 1995 to 2009. Purple: SIRs identified regardless of speed limit; red: SIRs with fastest speed faster than $450 \mathrm{~km} \mathrm{~s}^{-1}$; blue: SIRs with fastest speed faster than $500 \mathrm{~km} \mathrm{~s}^{-1}$; green: SIRs with fastest speed faster than $500 \mathrm{~km} \mathrm{~s}^{-1}$ and speed increase across the SIR more than $150 \mathrm{~km} \mathrm{~s}^{-1}$.

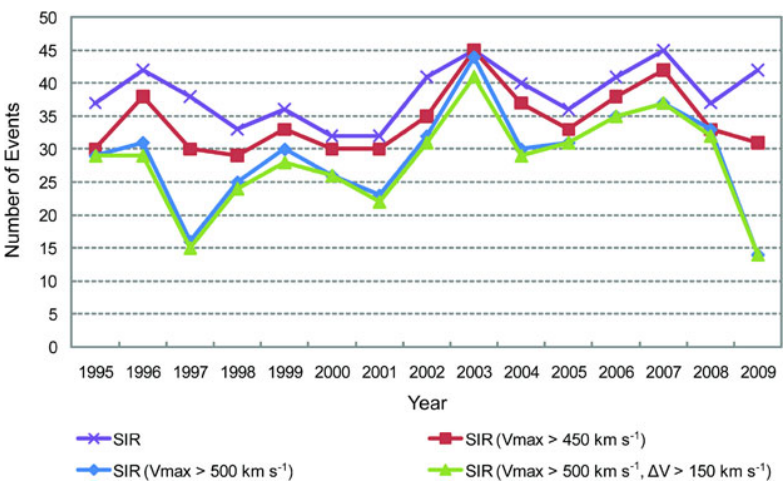

differently in each year, as illustrated in Figure 9. If we require the fastest speed to be above $500 \mathrm{~km} \mathrm{~s}^{-1}$ and the speed increase across the interaction region to be more than $150 \mathrm{~km} \mathrm{~s}^{-1}$, the SIR number (the green line in Figure 9) will decrease dramatically in 1997 and 2009. In fact, more than a half of the SIRs in 2009 do not have fast wind faster than $500 \mathrm{~km} \mathrm{~s}^{-1}$. Adding the two additional requirements, the SIR occurrence rate becomes more solar cycle dependent. SIRs occur more often in the declining phase, and 25 events occur during solar minimum 23/24, slightly less often than the 29 events during minimum 22/23. 
Table 3 Comparison of ICMEs between solar minima 22/23 and 23/24.

\begin{tabular}{llllllllllll}
\hline Solar & ICME & Shock & Dura- & Size \\
Min & $\begin{array}{l}\text { Num- } \\
\text { ber }\end{array}$ & $\begin{array}{l}\text { Rate } \\
(\%)\end{array}$ & $\begin{array}{l}\text { tion } \\
\text { (hour })\end{array}$ & $(\mathrm{AU})$ & $\begin{array}{l}V_{\max } \\
\left(\mathrm{km} \mathrm{s}^{-1}\right)\end{array}$ & $\begin{array}{l}V_{\min } \\
\left(\mathrm{km} \mathrm{s}^{-1}\right)\end{array}$ & $\begin{array}{l}V_{\text {mean }} \\
\left(\mathrm{km} \mathrm{s}^{-1}\right)\end{array}$ & $\begin{array}{l}V_{\text {exp }} \\
\left(\mathrm{km} \mathrm{s}^{-1}\right)\end{array}$ & $\begin{array}{l}B_{\max } \\
(\mathrm{nT})\end{array}$ & $\begin{array}{l}P_{\mathrm{t} \max } \\
(\mathrm{pPa})\end{array}$ & $\begin{array}{l}P_{\text {dynmax }} \\
(\mathrm{nPa})\end{array}$ \\
\hline $22 / 23$ & 4 & 0 & $32 \pm 5$ & $0.27 \pm 0.05$ & $388 \pm 12$ & $331 \pm 7$ & $360 \pm 6$ & $57 \pm 15$ & $13.0 \pm 1.7$ & $115 \pm 31$ & $7.2 \pm 1.5$ \\
$23 / 24$ & 8 & 25 & $21 \pm 3$ & $0.19 \pm 0.03$ & $413 \pm 14$ & $346 \pm 9$ & $379 \pm 11$ & $68 \pm 10$ & $10.1 \pm 1.4$ & $79 \pm 20$ & $4.4 \pm 1.0$ \\
\hline
\end{tabular}

\subsection{Interplanetary CMEs: Solar Minima $22 / 23$ vs. $23 / 24$}

In Figures 6 and 7, we mark the ICME intervals using the red bars. In total, only four ICMEs occurred during 1996, and eight ICMEs occurred during July 2008 - June 2009. About half of them occurred in near contact with SIRs, which is not surprising because the CMEs are often ejected from the streamer belts (Gosling, 1990). Table 3 lists the average properties of the ICMEs during the two solar minima. From the average values, in comparison with the last solar minimum, the ICMEs during the minimum 23/24 are shorter, smaller and slightly faster, while the maxima of magnetic field, total pressure, and dynamic pressure of the ICMEs during this minimum are all weaker. Because of the small number of events, the probable errors of the mean are not small relative to the mean values. If we consider these probable errors of the mean, the differences between the two solar minima are not that significant. Overall, the ICME features are similar during the two minima, except for a shorter duration and weaker dynamic pressure during the minimum 23/24.

Figure 10 shows the variations of the average ICME properties from 1995 to 2009. The annual ICME number varies from 3 to 40, in phase with the solar activity. The other ICME features, including the size, mean speed, expansion speed (approximated by $V_{\max }-V_{\min }$; there are studies using $\left(V_{\max }-V_{\min }\right) / 2$ as the expansion speed too), maximum $P_{\mathrm{t}}$, and maximum dynamic pressure, nearly all follow the solar activity. The annual average sizes of ICMEs over 1998-2005 are above the median value (red dashed line). In contrast to 2009 being the weakest year for SIRs, ICMEs have the lowest total pressure and dynamic pressure in 2008, among the 15 years. As the activity of Solar Cycle 24 rose in late 2009, eight ICMEs occurred in the second half of 2009, and the ICME occurrence rate in Figure 10 shows a rising trend.

In our study of ICMEs, we find that the temporal profiles of total pressure of ICMEs could be roughly classified into three characteristic patterns. Corresponding to the Group 1, 2, and 3 ICMEs, the $P_{\mathrm{t}}$ profile following a shock and/or sheath enhancement (if they exist) has a central pressure maximum, a steady plateau, or a gradual decay, respectively, as illustrated in Figures 2-4 of Jian et al. (2006b). These patterns are consistent with a model in which each ICME has a central flux rope, and the three groups of $P_{\mathrm{t}}$ profiles are attributed to different approach distances to the center of the flux rope (Jian et al. 2005, 2006b, 2008). If the spacecraft penetrates the central flux rope, it will observe the signatures of magnetic clouds (MCs), such as the coherent internal magnetic field rotations through a relatively large angle and low $\beta$ (see, e.g., Burlaga et al., 1981; Klein and Burlaga, 1982; Lepping, Jones, and Burlaga, 1990). The high magnetic field at the center of the flux rope will contribute to building a central $P_{\mathrm{t}}$ maximum, and such a profile will be classified as Group 1. If the spacecraft passes far from the center of the flux rope, the $P_{\mathrm{t}}$ will have a sharp rise at the bow shock or front edge of the ICME and then decline monotonically. Such a $P_{\mathrm{t}}$ profile will be sorted as Group 3. Group 2 ICME is the intermediate case between the above two, where the spacecraft passes a part of the flux rope, but not the center. Some ICMEs 
Figure 10 The variations of the annual average ICME properties from 1995 to 2009. From top to bottom: (a) monthly SSN from NOAA, (b) ICME number, (c) size approximated by the product of duration and mean speed, (d) mean solar wind speed of an ICME, (e) expansion speed of an ICME, (f) maximum total pressure, (g) maximum dynamic pressure. The error bar indicates the probable error of the mean. The red dashed line denotes the median value over all the ICMEs of the 15 years, while the green dotted line indicates the 15-year average value. Extended after Jian et al. (2006b).
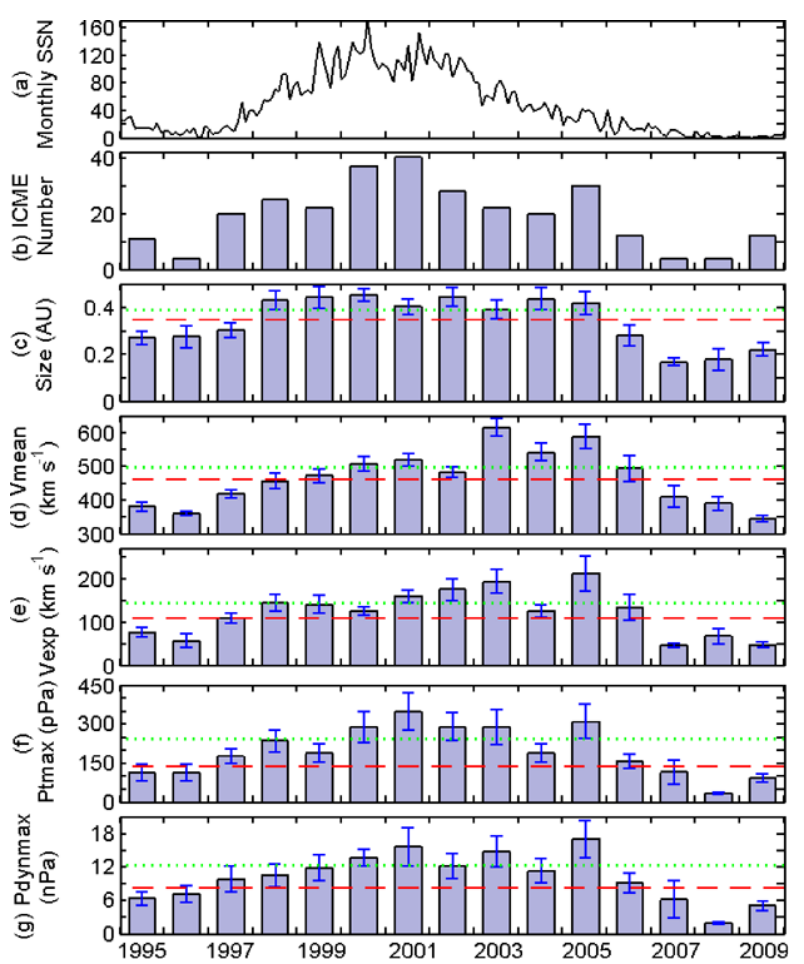

have irregular $P_{\mathrm{t}}$ profiles which are difficult to sort into any of the three groups. These are typically due to interactions with nearby SIRs or other transient structures, or because of complicated solar imprints.

Figure 11 illustrates the variations of the fractional occurrence rates of ICMEs in three groups and an unclassifiable category over 1995-2009. The fractional occurrence rate of Group 1 ICMEs varies in anti-phase with solar activity, with a peak during solar minima, opposite to the Group 3 ICMEs. This is consistent with the solar cycle variations of the MC fraction obtained by Richardson and Cane (2010). The higher fraction of Group 1 and 2 ICMEs agrees with the probability of encountering the central flux rope decreasing with solar activity, which is attributed to several factors (see, e.g., Jian et al., 2006b; Riley et al., 2006). First, CMEs near solar minimum are weaker and less dynamic, as demonstrated by smaller size and $P_{\mathrm{t} \max }$ shown in Figure 10, so they can disturb a smaller region around their embedded flux ropes. Second, CMEs during solar minimum occur in a smaller range of solar latitudes and they are closer to the equatorial plane. Third, the coronal structure and solar wind environment are less complicated during solar minimum. The fractional occurrence rate of unclassifiable ICMEs is roughly higher when the Sun is more active. In contrast with no unclassifiable ICMEs during solar minimum 22/23, there are two unclassifiable ICMEs during solar minimum 23/24, both with weak $P_{\mathrm{t}}$. Another reason of the unclassifiable ICMEs during solar minimum is that these ICMEs are often slow and sometimes overtaken by the following fast wind. In this situation, the density and magnetic field can be compressed and increase at the trailing part of the ICME, resulting in an irregular $P_{\mathrm{t}}$ profile, which is difficult to sort into any of the three groups. These are demonstrated by several examples in Kilpua et al. (2010). 
Figure 11 The variations of the fractional occurrence rates of ICMEs in three groups and unclassifiable category from 1995 to 2009 . The three groups are sorted based on the temporal profile of the total pressure. If an ICME has irregular profile of total pressure and is difficult to sort into any of the three groups, it is unclassifiable. Extended after Jian et al. (2008).

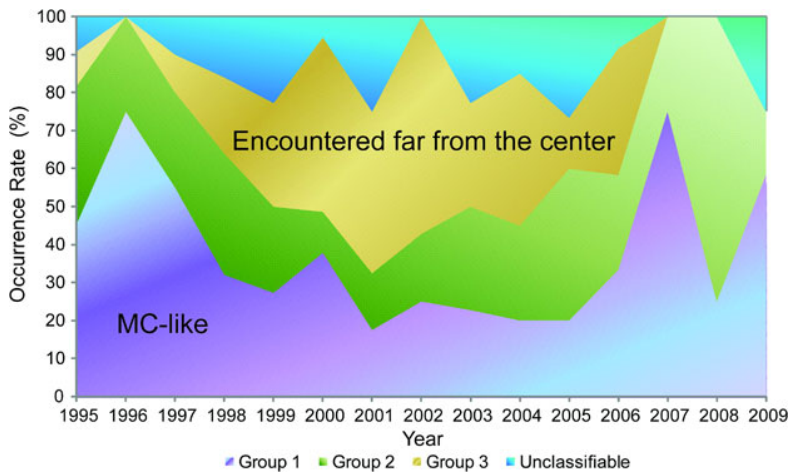

\subsection{Interplanetary Shocks: Solar Minima $22 / 23$ vs. $23 / 24$}

Lastly, we compare the interplanetary shocks during the solar minima 22/23 and 23/24. Figure 12 illustrates the solar cycle variations of shocks from 1995 to 2009 , with dark gray denoting the SIR-driven shocks, and light gray representing ICME-driven shocks. The event number and occurrence rate of ICME-driven shocks roughly follow the solar activity, while the event number and occurrence rate of SIR-driven shocks have no clear solar cycle dependence. Using the change of the total pressure across a shock as the proxy shock strength, we can see that the strength of both the SIR- and ICME-driven shocks varies in phase with the solar activity, being stronger around solar maximum and significantly weaker around solar minima, except 2001 for SIR-driven shock and 1999 for ICME-driven shock.

As listed in Tables 2 and 3, the shock rate of SIRs during this minimum is about $39 \%$, more than two times higher than during the previous minimum. There were no ICME-driven shocks in 1996, while two ICMEs $(25 \%)$ during this minimum drove shocks, so the shock rate of ICMEs during this minimum is higher than during the previous minimum. This is also illustrated in Figure 12. The higher occurrence rate of interplanetary shocks during solar minimum 23/24 is probably due to a slower fast magnetosonic speed of the solar wind, as discussed in Section 2.2. For the similar amount of force and speed increase, it is easier to form shocks during this solar minimum than during the previous minimum. In addition, as shown in Figure 12(c), although shocks occur more often, they are generally weaker during this minimum than during the previous minimum, probably because the shock divers, SIRs and ICMEs, are weaker during this minimum.

\section{Summary}

We have compared the various characteristics of solar minimum 23/24 in the context of the solar and solar wind observations available from the beginning of the space age. We have also compared the large-scale solar wind structures of this minimum with those of the last minimum. In contrast with previous minima in the space age, solar minimum 23/24 has the smallest SSN, slightly smaller solar radio flux, much weaker solar and interplanetary magnetic field, and a more warped coronal field neutral line and HCS. The solar wind during this minimum is slower, less dense, and cooler. The solar wind dynamic pressure and dawndusk electric field are weaker, resulting in much less geomagnetic activity. Associated with the weak IMF and quiet solar activity, the GCR reaches space-age record high, while there are no strong energetic proton events or Forbush decrease events during this solar minimum. 
Figure 12 The variations of interplanetary shocks driven by SIRs (dark gray) or ICMEs (light gray) from 1995 to 2009 . From top to bottom: (a) annual shock number, (b) shock association rates for SIRs and ICMEs, (c) absolute change of the total pressure across the shock, as the proxy indicator of the shock strength.
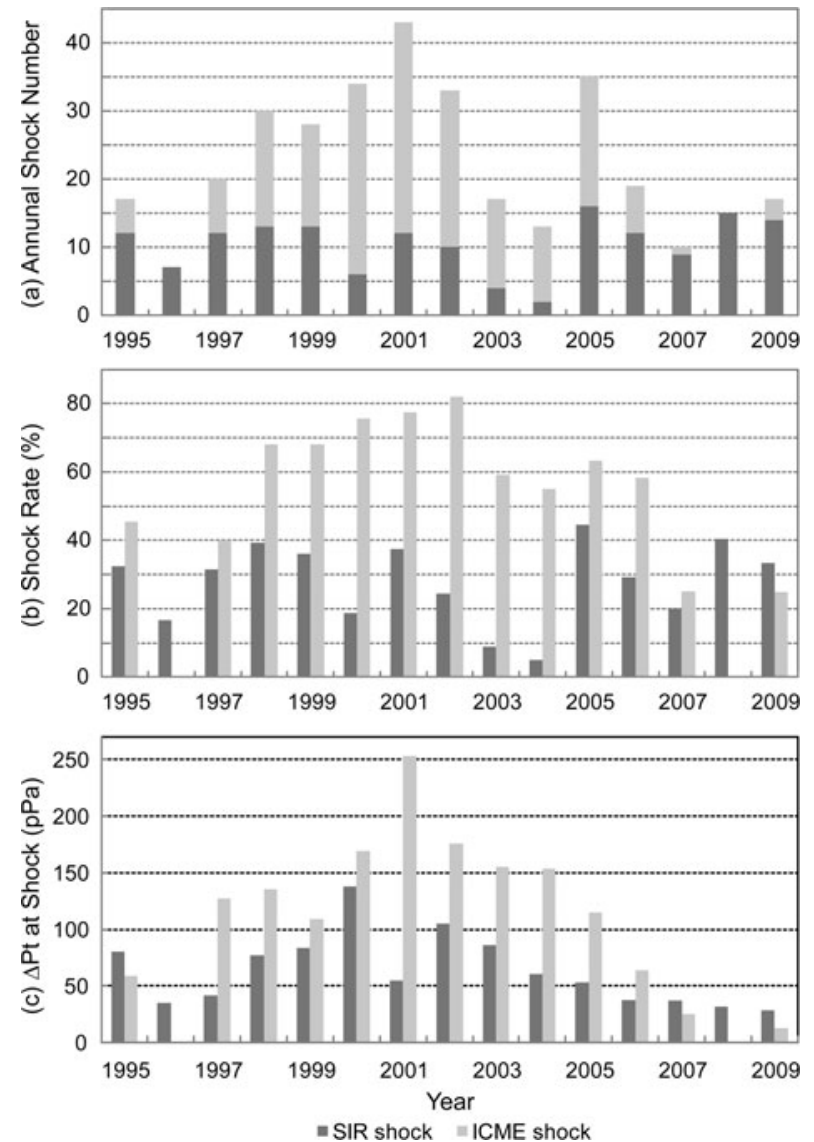

Based on the record of solar wind and geomagnetic activity over Solar Cycles 20-23, we also find the solar radiation flux, solar wind proton number density and dynamic pressure, as well as IMF are stronger in Solar Cycles 21 and 22, which have more sunspots. This is probably because the solar cycles with more sunspot emergence are usually more active and the solar field is stronger too. As a result, the geomagnetic activity is stronger in these two cycles. However, the solar wind speed and temperature, as well as the energetic proton flux do not seem to be affected much by a strong or weak solar cycle. Such study needs to continue for the coming solar cycles to determine the long-term correlation among SSN, solar wind parameters, geomagnetic activity, and energetic particle behavior.

Associated with the weak polar field, the HCS flattens toward the low-latitude regions more slowly. Attributed to the wavy HCS geometry and various sources of fast wind, there are still many SIRs during this minimum and their recurrent rate is slightly higher than during the minimum 22/23. About a half of SIRs occur with a HCS crossing nearby during this minimum, and such a rate is moderately higher than the last minimum, but it is still lower than the 15 -year average of $57 \%$. The mean speed, speed enhancement, maximum $P_{\mathrm{t}}$ and maximum dynamic pressure of SIRs have weak solar cycle dependence and are generally smaller during solar minima. The SIRs during this minimum are slightly slower and weaker than during the previous minimum. If we require the fastest wind speed to be faster than $500 \mathrm{~km} \mathrm{~s}^{-1}$, the SIR event number of the minimum 23/24 drops by more than a half and 
becomes smaller than the previous minimum. The occurrence rate, size, mean speed, expansion speed, maximum $P_{\mathrm{t}}$ and maximum dynamic pressure of ICMEs vary approximately in phase with solar activity over 1995-2009. The fractional occurrence rate of Group 1 ICMEs varies in anti-phase with solar activity, suggesting a higher probability of penetrating the center of the flux rope during solar minima. Compared with solar minimum 22/23, ICMEs during this minimum occur slightly more often, but these ICMEs are noticeably smaller and weaker.

Although the Sun and the solar wind are much quieter during this solar minimum, the ordinary phenomena of SIRs and ICMEs still exist. Wang and Sheeley (2003) proposed that there were still SIRs even during the Maunder minimum. In contrast with the dramatic weakening of solar wind parameters listed in Table 1, the extent of the decreases in speed, magnetic field, and pressure of both SIRs and ICMEs in Tables 2 and 3 are smaller than those of the general solar wind. If we take the probable error of the mean into consideration, the properties of SIRs and ICMEs during this minimum do not differ significantly from those at the previous minimum. Interplanetary shocks driven by SIRs or ICMEs occur more often during this solar minimum than the previous minimum, and most of them are driven by SIRs. The higher occurrence rate of shocks is likely due to the slower fast magnetosonic speed of the solar wind. Using the change of the total pressure at a shock as the proxy indicator of the shock strength, we find that the shocks during solar minimum 23/24 are weaker than during the previous minimum, probably related to the fact that the SIRs and ICMEs are overall weaker during this minimum.

It has been predicted that Solar Cycle 24 would be a weak cycle, which could be similar to weak cycles of the past 100 years and perhaps even to the Dalton minimum in the early 1800s (Russell, Luhmann, and Jian, 2010). During this extended period of low SSN there should still be fast and slow solar wind, SIRs, ICMEs, and interplanetary shocks. However, based on our observations to date and our pre-space-age ground-based observations, space weather events should be fewer and the geomagnetic activity may be less than in previous cycles. The root cause of this behavior appears to be the weak photospheric magnetic field (see, e.g., Russell, Luhmann, and Jian, 2010) and until the solar dynamo begins delivering greater magnetic flux to the photosphere, a weaker and less active solar wind will persist. Those interested in long-term space-climate forecasting are advised to keep their eyes on the strength of the photospheric magnetic field as this and perhaps the next few cycles take place.

Acknowledgements This work is supported by NASA's STEREO program through Grant NAS5-03131 administered by University of California, Berkeley. The OMNI data are obtained from the GSFC/SPDF OMNIWeb interface at http://omniweb.gsfc.nasa.gov. We thank all the PIs who provided the data to the OMNI data set and all the people who constructed and maintained the OMNI data. We have used the Wind plasma and magnetic field data to identify large-scale solar wind structures. We thank the MIT and Goddard plasma team (A.J. Lazarus and K.W. Ogilvie), 3DP plasma team (R.P. Lin), and the magnetometer team (R.P. Lepping) for making these data available. We have incorporated ACE data in this study. We are grateful to the PIs of the plasma analyzer (D.J. McComas) and the magnetometer (C.W. Smith) for making these data publicly available. We acknowledge the Wilcox Solar Observatory for providing the data of solar polar field strength and the HCS inclination. We thank the cosmic ray station of the University of Oulu and Sodankyla Geophysical Observatory for providing the cosmic ray data. We thank Nick Arge for providing the coefficients needed for the PFSS model. We are grateful to the Australian Antarctic Data Center for providing the data base of cosmic ray GLE events.

Open Access This article is distributed under the terms of the Creative Commons Attribution Noncommercial License which permits any noncommercial use, distribution, and reproduction in any medium, provided the original author(s) and source are credited. 


\section{References}

Abramenko, V., Yurchyshyn, V., Linker, J., Mikić, Z., Luhmann, J., Lee, C.O.: 2010, Astrophys. J. $712,813$. doi:10.1088/0004-637X/712/2/813.

Altschuler, M.A., Newkirk, G. Jr.: 1969, Solar Phys. 9, 131.

Berdichevsky, D.B., Szabo, A., Lepping, R.P., Vinas, A.F., Mariani, F.: 2000, J. Geophys. Res. 105(A12), 27289.

Bisi, M.M., Jackson, B.V., Buffington, A., Clover, J.M., Hick, P.P., Tokumaru, M.: 2009, Solar Phys. 256, 201. doi:10.1007/s11207-009-9350-9.

Bisi, M.M., Jackson, B.V., Breen, A.R., Dorrian, G.D., Fallows, R.A., Clover, J.M., Hick, P.P.: 2010, Solar Phys. 265, 233. doi:10.1007/s11207-010-9594-4.

Burlaga, L.F.: 1991, In: Schwenn, R., Marsch, E. (eds.) Physics of the Inner Heliosphere 2, Springer, Berlin, $1-22$.

Burlaga, L.F., Sittler, E., Mariani, F., Schwenn, R.: 1981, J. Geophys. Res. 86, 6673.

Burton, R.K., McPherron, R.L., Russell, C.T.: 1975, J. Geophys. Res. 80, 4204.

Cranmer, S.R., Hoeksema, J.T., Kohl, J.L.: 2010, In: SOHO-23: Understanding a Peculiar Solar Minimum, Proceedings of SOHO 23 Workshop CS-428, Astron. Soc. Pac., San Francisco, 336.

Crooker, N.U., Lazarus, A.J., Lepping, R.P., Ogilvie, K.W., Steinberg, J.T., Szabo, A., Onsager, T.G.: 1996, Geophys. Res. Lett. 23, 1275.

Dungey, J.W.: 1961, Phys. Rev. Lett. 6, 47.

Gibson, S.E., Webb, D.F., Thompson, B.J.: 2010, In: Cranmer, S.R., Hoeksema, J.T., Kohl, J.L. (eds.) SOHO23: Understanding a Peculiar Solar Minimum CS-428, Astron Soc. Pac., San Francisco, 223.

Gibson, S.E., Kozyra, J.U., de Toma, G., Emery, B.A., Onsager, T., Thompson, B.J.: 2009, J. Geophys. Res. 114, A09105. doi:10.1029/2009JA014342.

Gosling, J.T.: 1990, In: Russell, C.T., Priest, E.R., Lee, L.C. (eds.) Physics of Magnetic Flux Ropes, Geophys. Monogr. Ser. 58, AGU, Washington, 343.

Gosling, J.T.: 1997, In: Crooker, N., Joselyn, J.A., Feynman, J. (eds.) Coronal Mass Ejections, Geophys. Monogr. Ser. 99, AGU, Washington, 9.

Gosling, J.T., Pizzo, V.J.: 1999, Space Sci. Rev. 89, 21.

Harvey, J.W., Hill, F., Hubbard, R.P., Kenndy, J.R., Leibacher, J.W., Pintar, J.A., Gilman, P.A., et al.: 1996, Science 272(5266), 1284. doi:10.1126/science.272.5266.1284.

Hoeksema, J.T.: 1995, Space Sci. Rev. 72, 137.

Hoeksema, J.T., Wilcox, J.M., Scherrer, P.H.: 1983, J. Geophys. Res. 88, 9910.

Hundhausen, A.J.: 1972, Coronal Expansion and Solar Wind, Springer, New York.

Jian, L., Russell, C.T., Gosling, J.T., Luhmann, J.G.: 2005, In: Fleck, B., Zurbuchen, T.H., Lacoste, H. (eds.) Proc. Solar Wind 11/SOHO 16-Connecting Sun and Heliosphere SP-592, ESA, Noordwijk, 731.

Jian, L., Russell, C.T., Luhmann, J.G., Skoug, R.M.: 2006a, Solar Phys. 239, 337. doi:10.1007/ s11207-006-0132-3.

Jian, L., Russell, C.T., Luhmann, J.G., Skoug, R.M.: 2006b, Solar Phys. 239, 393. doi:10.1007/ s11207-006-0133-2.

Jian, L.K., Russell, C.T., Luhmann, J.G., Skoug, R.M., Steinberg, J.T.: 2008, Solar Phys. $249,85$. doi:10.1007/s11207-008-9161-4.

Kahler, S.W.: 2010, In: Cranmer, S.R., Hoeksema, J.T., Kohl, J.L. (eds.) SOHO-23: Understanding a Peculiar Solar Minimum CS-428, Astron. Soc. Pac., San Francisco, 259.

Kilpua, E.K.J., Jian, L.K., Li, Y., Luhmann, J.G., Russell, C.T.: 2010, J. Atmos. Solar-Terr. Phys. submitted.

King, J.H., Papitashvilii, N.E.: 1994, Interplanetary Medium Data Book Supplement 5, 1988 - 1993, NASA NSSDC/WDC-A-R\&S, 94-08, Greenbelt.

King, J.H., Papitashvili, N.E.: 2005, J. Geophys. Res. 110, A02104. doi:10.1029/2004JA010649.

Kirk, M.S., Pesnell, W.D., Young, C.A., Hess Webber, S.A.: 2009, Solar Phys. 257, 99. doi:10.1007/ s11207-009-9369-y.

Klein, L.W., Burlaga, L.F.: 1982, J. Geophys. Res. 87, 613.

Kojima, M., Kakinuma, T.: 1987, J. Geophys. Res. 92, 7269.

Lee, C.O., Luhmann, J.G., Zhao, X.P., Liu, Y., Riley, P., Arge, C.N., Russell, C.T., de Pater, I.: 2009, Solar Phys. 256, 345. doi:10.1007/s11207-009-9345-6.

Leibacher, J.W.: 1999, Adv. Space Res. 24, 173.

Lepping, R.P., Jones, J.A., Burlaga, L.F.: 1990, J. Geophys. Res. 95, 11957.

Lepping, R.P., Acũna, M.H., Burlaga, L.F., Farrell, W.M., Slavin, J.A., Schatten, K.H., Mariani, F., et al.: 1995, Space Sci. Rev. 71, 207.

Lin, R.P., Anderson, K.A., Ashford, S., Carlson, C., Curtis, D., Ergun, R., Larson, D., et al.: 1995, Space Sci. Rev. 71, 125. 
Luhmann, J.G., Lee, C.O., Li, Y., Arge, C.N., Galvin, A.B., Simunac, K., Russell, C.T., et al.: 2009, Solar Phys. 256, 285. doi:10.1007/s11207-009-09354-5.

Manoharan, P.K.: 2009, In: Kosovichev, A.G., Andrei, A.H., Rozelot, J.-P. (eds.) Proc. IAU Symposium, Solar and Stellar Variability: Impact on Earth and Planets 24, 356. doi:10.1017/S1743921309992912.

Manoharan, P.K.: 2010, In: Corbett, I.F. (ed.) Highlights of Astronomy, 15, 484. doi:10.1017/ S1743921310010343.

McComas, D.J., Bame, S.J., Barker, P., Feldman, W.C., Phillips, J.L., Riley, P., Griffee, J.W.: 1998, Space Sci. Rev. 86, 563.

McComas, D.J., Ebert, R.W., Elliott, H.A., Goldstein, B.E., Gosling, J.T., Schwadron, N.A., Skoug, R.M.: 2008, Geophys. Res. Lett. 35, L18103. doi:10.1029/2008GL034896.

McDonald, F.B., Webber, W.R., Reames, D.V.: 2010, Geophys. Res. Lett. 37, L18101. doi:10.1029/ 2010 GL044218.

Mewaldt, R.A., Davis, A.J., Lave, K.A., Leske, R.A., Stone, E.C., Wiedenbeck, M.E., Binns, W.R., et al.: 2010, Astrophys. J. 723, L1. doi:10.1088/2041-8205/723/1/L1.

Murdin, P.: 2000, In: Murdin, P. (ed.) Encyclopedia of Astronomy and Astrophysics, 4331. doi:10.1888/ $0333750888 / 4331$.

Ogilvie, K.W., Chornay, D.J., Fritzenreiter, R.J., Hunsaker, F., Keller, J., Lobell, J., Miller, G., et al.: 1995, Space Sci. Rev. 71, 55.

Oh, S.Y., Yi, Y.: 2009, J. Geophys. Res. 114, A11102. doi:10.1029/2009JA014190.

Palmroth, M., Pulkkinen, T.I., Janhunen, P., McComas, D.J., Smith, C.W., Koskinen, H.E.J.: 2004, J. Geophys. Res. 109, A11302. doi:10.1029/2004JA010529.

Perreault, P., Akasofu, S.-I.: 1978, Geophys. J. Roy. Astron. Soc. 54, 547.

Richardson, I.G., Cane, H.V.: 2010, Solar Phys. 264, 189. doi:10.1007/s11207-010-9568-6.

Riley, P., Schatzman, C., Cane, H.V., Richardson, I.G., Gopalswamy, N.: 2006, Astrophys. J. 647, 648.

Rosenberg, R.L., Coleman, P.J. Jr.: 1969, J. Geophys. Res. 74(24), 5611.

Russell, C.T., Shinde, A.A., Jian, L.: 2005, Adv. Space Res. 35, 2178.

Russell, C.T., Luhmann, J.G., Jian, L.K.: 2010, Rev. Geophys. 48, RG2004. doi:10.1029/2009RG000316.

Schatten, K.H., Wilcox, J.M., Ness, N.F.: 1969, Solar Phys. 6, 442.

Sheeley, N.R. Jr.: 2010, In: Cranmer, S.R., Hoeksema, J.T., Kohl, J.L. (eds.) SOHO-23: Understanding a Peculiar Solar Minimum CS-428, Astron. Soc. Pac., San Francisco, 3.

Smith, C.W., L'Heureux, J., Ness, N.F., Acuña, M.H., Burlaga, L.F., Scheifele, J.: 1998, Space Sci. Rev. 86, 613.

Smith, E.J., Balogh, A.: 2008, Geophys. Res. Lett. 35. doi:10.1029/2008GL035345.

Svalgaard, L., Duvall, T.L. Jr., Scherrer, P.H.: 1978, Solar Phys. 58, 225.

Tokumaru, M., Kojima, M., Fujiki, K.: 2010, J. Geophys. Res. 115, A04102. doi:10.1029/2009JA014628.

Tokumaru, M., Kojima, M., Fujiki, K., Hayashi, K.: 2009, Geophys. Res. Lett. 36, L09101. doi:10.1029/2009GL037461.

Ulrich, R.K., Evans, S., Boyden, J.E., Webster, L.: 2002, Astrophys. J. Suppl. 139, 259.

Wang, Y.-M., Sheeley, N.R. Jr.: 2003, Astrophys. J. 591, 1248.

Wang, Y.-M., Robbrecht, E., Rouillard, A.P., Sheeley, N.R. Jr., Thernisien, A.F.R.: 2010, Astrophys. J. 715, 39. doi:10.1088/004-637X/715/1/39.

Zhang, T.L., Delva, M., Baumjohann, W., Auster, H.U., Carr, C., Russell, C.T., Barabash, S., et al.: 2007 , Nature 450, 654. doi:10.1038/nature06026.

Zurbuchen, T.H., Richardson, I.G.: 2006, Space Sci. Rev. 123, 31. doi:10.1007/s11214-006-9010-4. 